\title{
Die Conchoidenfläche, eine Linienfläche 4. Ordnung.
}

Von G. Huber in Bern.

\section{Definition und Erzeugung der Fläche durch Bewegung einer Geraden.}

Zieht man vom Nullpunkt $O$ eines rechtwinkligen, ebenen Coordinatensystems Strahlen nach einer festen Geraden, $y=p$, der Leitlinie $L$, und trägt auf jedem Strahl vom Schnittpunkt mit $L$ aus nach beiden Seiten eine beliebig gewählte, aber constante Strecke $s$ ab, so liegen alle so erhaltenen Endpunkte aut einer Curve 4. Ordnung, welche die Conchoide des Nikomedes heißt. Ihre Gleichung ist:

$$
(y-p)^{2}\left(x^{2}+y^{2}\right)=s^{2} y^{2}
$$

Die Curve besteht aus zwei unendlichen Zweigen, welche die Leitlinie $L$ zur gemeinschaftlichen Asymptote haben, deren unendlich ferner Berührungspunkt ein Selbstberührungspunkt der Curve ist. Der Nullpunkt $O$ ist conjugierter Pankt, Spitze oder Knotenpunkt, jenachdem $s \lesseqgtr p$ ist. Bei veränderlichem $s$ erbält man unendlich viele Conchoiden von derselben Asymptote $L$ und gemeinschaftlichem Doppelpunkt $O$ und zwar besteht die Curve:

Für $s=o$ aus der doppelten Leitlinie $L$ und dem conjugierten Punkt $O$.

Für $s<p$ aus zwei unendlichen, einfachen $Z w e i g e n$, zu verschiedenen Seiten von $L$ und dem conjugierten Punkt $O$.

Für $s=p$ aus zwei unendlichen, einfachen Zweigen, der eine mit Spitze in $O$.

Für $s>p$ aus zwei unendlichen Zweigen, der eine mit Schleife und Doppelpunkt in $O$.

Für $s=\infty$ aus der doppelt gelegten $x$ Axe und einem Kreis von unendlich großem Radius um $O$ als Mittelpunkt.

Der Ort der Wendepunkte aller Conchoiden dieses Systems ist die $\mathrm{Neil}$ 's che Parabel $y^{3}=2 p x^{2}$. 
Wir setzen nun den veränderlichen Parameter $s$ gleich der räumlichen Coordinate $z$, dann werden die Conchoiden des obigen Systems aus einander herausgehoben, sie liegen in Ebenen parallel zur $(x y)$ Ebene, oberhalb und unterhalb derselben und bilden in ihrer G es a m m the i teine Fläche, welche die Conchoid en fläch e heißen möge.

Die Fläche ist von der 4. Ordnung, ihre Gleichung heißt:

$$
(y-p)^{2}\left(x^{2}+y^{2}\right)=y^{2} z^{2} \text {. }
$$

Aus dieser Definition ergeben sich sofort folgende Eigenschaften:

1. Die Fläche liegt symmetrisch zur $(x y)$ und zur $(y z)$ Ebene.

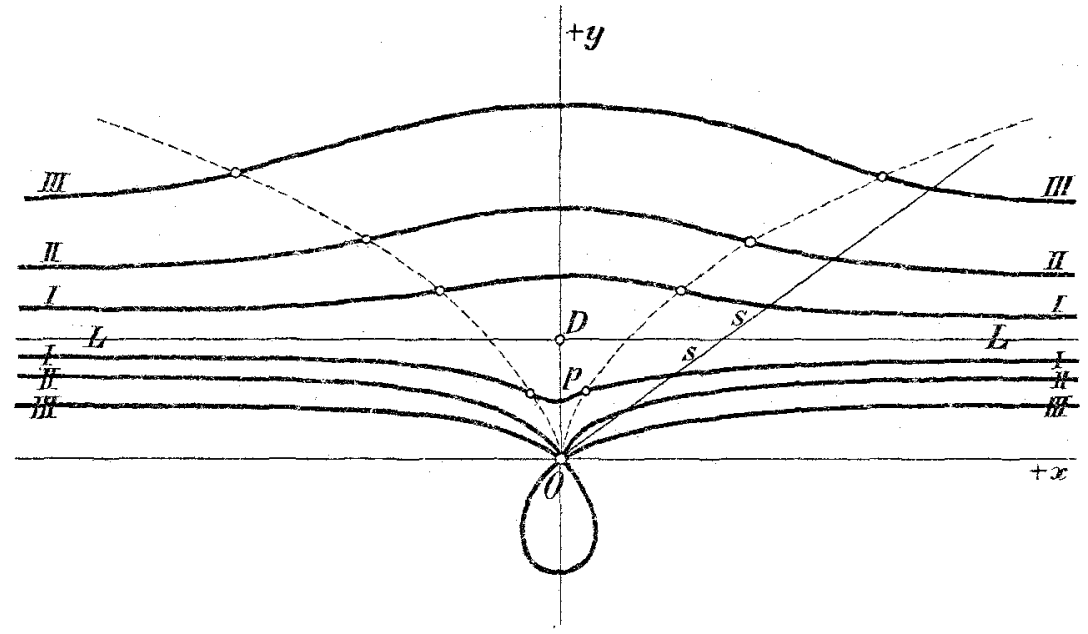

Fig. 1. Conchoidensystem.

2. Die zur $(x y)$ Ebene parallelen ebenen Schnitte $z= \pm s$, sind die oben angegebenen Conchoiden der verschiedenen Arten; $z= \pm p$ gibt zwei Conchoiden mit Spitzen auf der $z$-Axe.

3. Die Leitlinie $L(y=p)$ in der $(x y)$ Ebene ist Doppelgerade $L$ der Fläche.

4. Die $z$-Axe, als Ort aller Doppelpunkte der Conchoiden $z=s>p$ ist ebenfalls Doppelgerade, $Z$, der Fläche. Die Punkte $z= \pm p$ auf derselben, die wir mit $S$ und $S^{\prime}$ bezeichnen, sind uniplanare Doppelpunkte, mit der $(y z)$ Ebene als gemeinschaftlicher Tangentialebene. Das zwischen $S$ und $S^{\prime}$ liegende Stück dieser Doppelgeraden, den Werten $z=s<p$ entsprechend, ist is oliert und liegt nicht auf der Fläche selbst.

5. Die zur $(x y)$ Ebene parallele unendlich ferne Ebene schneidet die Fläche in einem $\mathrm{Kr}$ eis von unendlich großem Radius, dessen Mittelpunkt im unendlich fernen Punkt der $z$-Axe 
liegt und dessen Richtungskegel die Gleichung $x^{2}+y^{2}=z^{2}$ hat, und in der doppelt gelegten unendlich fernen Geraden der $(x y)$ Ebene, es ist dies eine dritte $D$ op pelgerade, $G_{\infty}$, der Fläche.

6. Die durch die Doppelgerade $L \mathrm{~h}$ ind u $\mathrm{ch}$ gehende, zur $(x z)$ Ebene parallele Ebene $y=p$, ist A s y m p to te n e b e n e der Fläche, längs ihrer unendlich fernen Geraden findet Selbstberührung der Fläche statt, diese Berührungsgerade fällt zusammen mit der obigen Doppelgeraden $G_{\infty} \operatorname{der}(x z)$ Ebene.

Ebenenbüschel durch die Doppelgerade $G_{\infty}$.

Eine zur $(x z)$ Ebene parallele Ebene, $y=b$, schneidet die Fläche, nebst in $G_{\infty}$, noch in der Hyperbel:

$$
\frac{z^{\prime 2}}{(b-p)^{2}}-\frac{x^{\prime 2}}{b^{2}}=1
$$

deren reelle Axe in der Richtung der $z$-Axe liegt. Für $b=0$ reduciert sich die Hyperbel auf die Doppelgerade $Z$, für $b=p$ auf $L$, and für $b=\frac{p}{2}$ entsteht die gleichseitige Hyperbel $z^{\prime 2}-x^{\prime 2}=\frac{p^{2}}{4}$. Für $b=\infty$ werden beide Axen der Hyperbel unendlich groß.

Die zur $(y z)$ Ebene parallelen ebenen Schnitte sind Curven 4. Ordnung mit Doppelpunkt in $L$ und $G_{\infty}$. Die $(y z)$ Ebene selbst schneidet die Fläche in den beiden Geraden:

$$
y= \pm z+p
$$

sie gehen durch den Schnittpunkt $D$ der Leitlinie $L$ mit der $+y$ Axe, bilden mit der $(x y)$ Ebene die Winkel $\pm 45^{\circ}$ und gehen durch die Doppelpunkte $S$ und $S^{\prime}$ auf der $z$-Axe. (Fig. 2).

Ebenenbüschel durch die Doppelgerade $Z$.

Jede Ebene durch eine Doppelgerade schneidet die Fläche noch in einer Curve 2. Ordnung. Die Gleichung einer beliebigen Schmittebene durch die $z$-Axe ist $y=\operatorname{tg} \alpha . x$, wo $a$ der Winkel ist, den die Ebene mit der positiven $(x z)$ Ebene bildet. Macht man diese Ebene durch Drehung des Coordinatensystems um den Winkel $\alpha$ um die z-Axe zur $(x z)$ Ebene und setzt in der transformierten Gleichung $y^{\prime}=0$, so erhält man als Schnittcurve die beiden Geraden:

$$
z= \pm\left(x^{\prime}-\frac{p}{\sin \alpha}\right)
$$

welche für jedes $a$ reell sind. Für $\alpha=0$ fallen beide Geraden mit $G_{\infty}$ zusammen und für $\alpha= \pm \frac{\pi}{2}$ sind sie die beiden Geraden $D S$ und $D S^{\prime}$ in der $(y z)$ Ebene. Die Abschnitte der Geraden 


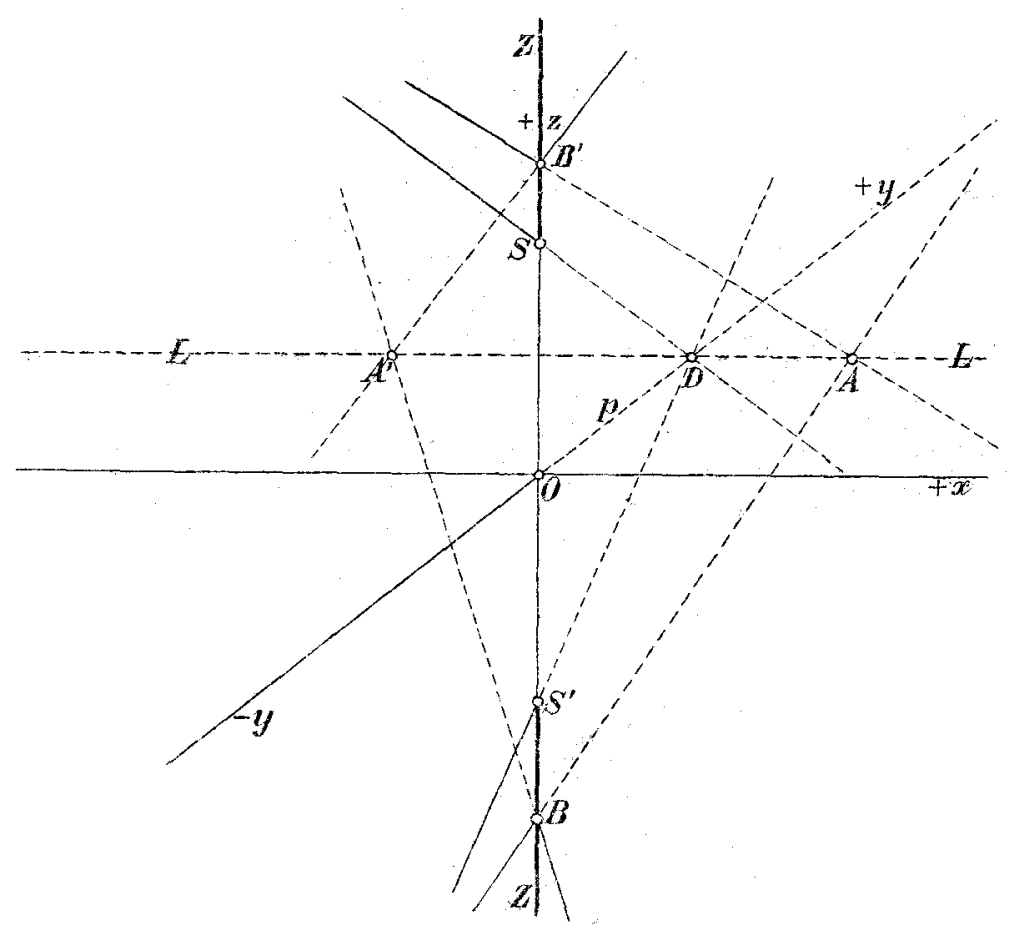

Fig. 2. Conchoidenfläche.

auf der $z$-Axe, der Doppelgeraden $Z$, sind $z_{0}= \pm \frac{p}{\sin \alpha}$, beide gehen durch denselben Punkt $A\left(x_{0}^{\prime}=\frac{p}{\sin \alpha}\right)$ der $x^{\prime}$-Axe; welcher der Schnittpunkt der Schnittebene mit $L$ ist. (Fig. 2). Beide Gerade bilden sowohl mit der $(x y)$ Ebene als auch mit $Z$ die Winkel $\pm 45^{\circ}$. Es gilt also der Satz:

Durch jeden Punkt der Doppelgeraden $L$ gehen zwei Gerade der Fläche, welche mit der $(x y)$ Ebene die Winkel $\pm 45^{\circ}$ bilden und welche die Doppelgerade $Z$ in gleichem Abstande von Nullpunkt unter dem Winkel $45^{\circ}$ schneiden. Die Fußpunkte jeder Geraden auf $L$ und $Z$ sind yon $O$ gleich weit entfernt. Diese Geradenpaare werden auf der Fläche von dem Ebenenbüschel durch $Z$ ausgeschnitten.

Ebenenbüschel durch die Doppelgerade $L$.

Wir machen zuerst die Leitlinie $L$ in der $(x y)$ Ebene durch Parallelverschiebung des Axensystemes $\left(y=y^{\prime}+p\right)$ zur $x^{\prime}$-Achse, so dass $D$ zum Nullpunkt und die Asymptotenebene $y=p$ zur $\left(x^{\prime} z^{\prime}\right)$ Ebene wird. Die Gleichung einer beliebigen Ebene durch $L$ ist $y^{\prime}=\operatorname{tg} \beta . z^{\prime}$, wo $\beta$ ihr Neigungswinkel mit der Asymptotenebene ist. Wir drehen dann das Coordinatensystem um den Winkel 
$\beta$ um $L$, so dass die Schnittebene zur $\left(x^{\prime} z^{\prime}\right)$ Ebene wird und setzen dann $y^{\prime}=0$. Die Gleichung der Schnittcurve auf der Fläche in dieser Ebene wird:

$$
x^{\prime}= \pm \sqrt{\cos 2 \beta}\left(z^{\prime}+\frac{p}{\sin \beta}\right) \text {. }
$$

Es sind dies zwei Gerade, welche durch den Punkt $z^{\prime}=-$ $-\frac{p}{\sin \beta}$ der $z^{\prime}$-Axe hindurchgehen, es ist der Schnittpunkt $B$ der Schnittebene $(\beta)$ mit der ursprünglichen $z$-Axe, der Doppelgeraden $Z$. Die beiden Geraden schneiden $L$ in gleichem Abstande $x^{\prime}=$ $= \pm p \frac{\sqrt{\cos 2} \beta}{\sin \beta}$ von $D$ in $A$, und $A^{\prime}$ (Fig. 2) und haben gleiche Neigung gegen $L$; sie sind nur reell, wenn $\beta$ absolut $<45^{\circ}$ ist, d. h. wenn die Schnittebene gegen die Asymptotenebene beiderseits weniger als $45^{\circ}$ geneigt ist. Für $\beta= \pm 45^{\circ}$ fallen sie in $x^{\prime}=0$, mit der $z^{\prime}$-Axe zus a mmen, der Axenschnitt auf $Z$ wird $z= \pm p$, es sind dies bezüglich die Geraden $D S^{\prime}$ und $D S$ in $\operatorname{der}(y z)$ Ebene, dies sind Grenzlagen der Geraden; $z$ w is ch en $S$ und $S^{\prime}$ auf $Z$ liegen keine Schnittpunkte ron reellen Geraden der Fläche. Es gilt also der Satz:

Durch jeden Punkt der Doppelgeraden Z, mit Ausnahme der Punkte zwischen den uniplanaren Doppelpunkten $S$ und $S^{t}$ $(z= \pm p)$, gehen zwei Gerade der Fläche, welche $L$ in gleichem Abstande von $D$ schneiden. Diese Geradenpaare liegen in Ebenen, die alle durch die Doppelgerade $L$ gehen. Zwischen $S$ und $S^{\prime}$ ist die Doppelgerade eine isolierte Gerade der Fläche.

Aus diesen beiden Sätzen folgt:

Die Conchoidenfläche kann erzengt werden durch B e w e g ung einer Geraden, Erzeagenden, die längs zweier fester, windschiefer und zu einander senkrechter Geraden, Leitlinien, $L$ und $Z$, so fortgeleitet, dass sie mit der einen Leitlinie $Z$ and mit der durch die andere, $L$, senkrecht zur ersten gelegten Ebene, stets einen Winkel von $45^{\circ}$ bildet; oder auch so, dass die Schnittpunkte der bewegten Geraden mit $L$ und $Z$ stets gleichen Abstand haben vom Fußpunkt $O$ der einen Leitlinie $Z$, in der durch die andere Leitlinie $L$ senkrecht zur ersteren gelegten Ebene. Di e Conchoidenfläche ist also eine windschiefe Regelfläche 4. Ordnung.

Zieht man durch den Nullpunkt zu sämmtlichen Erzeugenden der Fläche parallele Gerade, so bilden diese den Richtungsoder Leitkegel der Fläche. Weil nun alle Erzeugenden die $z$-Axe unter dem Winkel $45^{0}$ schneiden, so ist der Leitkegel ein gerader Kreiskegel vom Öffnungswinkel. $90^{\circ}$, dessen Axein der $z$-Axe liegt; seine Gleichung ist:

$$
x^{2}+y^{2}=z^{2} \text {. }
$$


Die Erzeugenden dieses Kegels schneiden die entsprechenden der Fläche im Unendlichen in einem Kreis von unendlich großem Radius, der in einer zur z-Axe senkrechten Ebene um den unendlich fernen Punkt derselben beschrieben ist. Die Fläche lässt sich nun auch folgenderweise definieren:

Die Conchoidenfläche ist eine Regelfläche, erzeugt durch Bewegung einer Geraden, die stets drei ebene Leitlinien schneidet. Zwei derselben sind zwei zu einander senkrechte, windschiefe Gerade, während die dritte Leitlinie ein um den unendlich fernen Punkt der einen Leitgeraden mit unendlich großem Radius beschriebener Kreis ist, dessen Ebene zu dieser senkrecht steht. Die in dieser unendlich fernen Ebene liegende Erzeugende $G_{\infty}$ ist Doppelerzeugende der Fläche. Durch jeden Punkt der zwei Leitgeraden gehen zwei Erzengende, sie sind Doppelcurven der Fläche, während durch jeden Punkt des unendlich fernen Kreises nur eine Erzeugende geht, sonst durchschneiden sich keine zwei Erzeugende der Fläche.

Der Leitkegel schneidet die Conchoidenfläche außerdem noch in der gleichseitigen Hyperbel:

$$
z^{\prime 2}-x^{\prime 2}=\frac{p^{2}}{4}
$$

welche in der Ebene $y=\frac{p}{2}$ liegt; ihre Axe liegt in der Richtung der $z$-Axe und ihre Asymptoten sind parallel den in der $(x z)$ Ebene liegenden Kegelerzeugenden.

Der Leitkegel schneidet die concentrische Einheitskugel $x^{2}+y^{2}+z^{2}=1$ in $z$ wei Kleinkreisen parallel der $(x y)$ Ebene vom Radius $r=\frac{1}{2} \sqrt{2}$, im Abstand $\frac{1}{2} \sqrt{2}$ von $\operatorname{der}(x y)$ Ebene, sie heißen die sphärische Indikatrix der Erzeugenden der Fläche.

\section{Parametercurven auf der Fläche.}

Die Coordinaten eines Conchoidenpunktes in der $(x y)$ Ebene lassen sich durch trigonometrische Functionen eines veränderlichen Parameters $v$ in der Form

$$
x=(p-s \cos v) \tan g, \quad y=(p-s \cos v)
$$

darstellen, wo $v$ der Winkel ist, den der Leitstrahl des Punktes mit der positiven $y$-Axe bildet. Setzt man die Strecke $s$ gleich einem verïnderlichen Parameter $u$, so lassen sich die Coordinaten der Punkte der Conchoidenfläche folgenderweise durch $\mathrm{krumm-}$ linige Coordinaten $u, v$ darstellen: 


$$
\begin{aligned}
& x=(p-u \cos v) \operatorname{tang} v \\
& y=(p-u \cos v) \\
& z=u
\end{aligned}
$$

wo $v$ der Winkel ist, den eine um die Doppelgerade $Z$ in negativer Richtung sich drehende Ebene mit der positiven $(y z)$ Ebene bildet und $u$ der Abstand einer zur $(x y)$ Ebene parallelen, veränderlichen Ebene von dieser Ebene.

Den Werten $u=p$ und $v=0$, bez. $2 \pi$ entspricht der Doppelpunkt $S(0,0, p)$.

Den Werten $u=-p, v= \pm \pi$ entspricht der Doppelpunkt $S^{\prime}(0,0,-p)$.

Die Parametercurven $u=$ konstant sind die Conchoiden, die Parametercurven $v=$ konstant sind die geradlinigen Erzeugenden der Fläche. Jedem Wert $v$ entspricht eine bestimmte Erzeugende. Durch jeden Punkt der zwei Leitlinien $L$ und $Z$ gehen je zwei Erzeugende entsprechend den Werten $v$ und $180^{\circ}+\dot{v}$ bezüglich $\pm v$. Für $v=0$ und $v=180^{\circ}$ erhält man die zwei Erzeugenden $x=0, y \pm z=p$, es sind die in der (yz) Ebene liegenden Erzeugenden $S \bar{D}$ und $S^{\prime} D$, welche sich in $D$ auf $L$ rechtwinklig durchschneiden.

Für $v= \pm \frac{\pi}{2}$ werden die Gleichungen der Erzeugenden :

$$
x= \pm \infty, y=p, z=u
$$

d. h. diese zwei Erzeugenden fallen mit der unendlich fernen Geraden der Asymptotenebene $y=p$ zusammen, sie bilden die Doppelgerade $G_{\infty}$. Die Erzeugenden schneiden die positive oder negative $z$-Axe, auberhalb der Strecke $S S^{\prime}$, jenachdem die Winkelwerte $v$ im ersten und vierten bezüglich im zweiten und dritten Quadranten liegen. $Z$ ist

Die Gleichung der Leitlinie $L$ ist $u=0$ und diejenige von

$$
u=\frac{p}{\cos v}
$$

denn für diesen Wert von $u$ wird $x=0, y=0$ und $z=\frac{p}{\cos v}=$ $=$ Abschnitt der Erzeugenden $v$ auf $Z$, dieser wird ein Minimum $z= \pm p$, für $v=0,2 \pi$ und $v= \pm \pi \mathrm{d}$. h. in den Punkten $S$ und $S^{\prime}$.

Wir bestimmen nun die Gaubischen Fundamentalgrößen. Es wird :

$$
\frac{d x}{d u}=-\sin v, \quad \frac{d y}{d u}=-\cos v, \quad \frac{d z}{d u}=1
$$




$$
\begin{aligned}
& \frac{d x}{d v}=\frac{p}{\cos ^{2} v}-u \cos v, \quad \frac{d y}{d v}=u \sin v, \quad \frac{d z}{d v}=0 \\
& \frac{d^{2} x}{d u^{2}}=\frac{d^{2} y}{d u^{2}}=\frac{d^{2} z}{d u^{2}}=0 \\
& \frac{d^{2} x}{d v^{2}}=2 p \frac{\operatorname{tg} v}{\cos ^{2} v}+u \sin v, \quad \frac{d^{2} y}{d v^{2}}=u \cos v, \quad \frac{d^{2} z}{d v^{2}}=0 \\
& \frac{d^{2} x}{d u d v}=-\cos v, \quad \frac{d^{2} y}{d u d v}=\sin v, \quad \frac{d^{2} z}{d u d v}=0 \text {. }
\end{aligned}
$$

Fundamentalgrößen erster Ordnung:

$$
\left\{\begin{array}{l}
E=\Sigma\left(\frac{d x}{d u}\right)^{2}=2 \\
F=\Sigma \frac{d x}{d u} \cdot \frac{d x}{d v}=-p \frac{\operatorname{tg} v}{\cos v} \\
G=\Sigma\left(\frac{d x}{d v}\right)^{2}=\left(p^{2}-2 p u \cos ^{3} v+u^{2} \cos ^{4} v\right): \cos ^{4} v \\
\delta^{2}=E G-F^{2}=\left\{p^{2} \operatorname{tg}^{2} v+2(p-u \cos v)^{2}\right\}: \cos ^{2} v .
\end{array}\right.
$$

Fundamentalgröben zweiter Ordnung:

$$
\begin{gathered}
D=\frac{1}{\delta}\left|\begin{array}{l}
\frac{d^{2} x}{d u^{2}}, \frac{d^{2} y}{d u^{2}}, \frac{d^{2} z}{d u^{2}} \\
\frac{d x}{d u}, \frac{d y}{d u}, \frac{d z}{d u} \\
\frac{d x}{d v}, \frac{d y}{d v}, \frac{d z}{d v}
\end{array}\right|=0, D^{\prime}=\frac{p}{\partial} \frac{\operatorname{tg} v}{\cos v}, \\
D^{\prime \prime}=\frac{u}{\delta \cos v}\left(p-u \cos v-2 p \operatorname{tg}^{2} v\right) .
\end{gathered}
$$

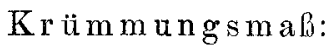

$$
k=\frac{1}{\rho_{1} \rho_{2}}=\frac{D \cdot D^{\prime \prime}-D^{\prime 2}}{\delta^{2}}=\frac{-p^{2} \sin ^{2} v}{\left[p^{2} \operatorname{tg}^{2} v+2(p-u \cos v)^{2}\right]^{2}} \text {. }
$$

Weil $k$ stets negativ ist, so sind alle Punkte der Fläche hyperbolische "Punkte, die Hauptkrümmungsradien $\rho_{1}$ und $\rho_{2}$ sind in jedem entgegengesetzt gerichtet.

Für $v=0, \pi$ und $\pm \frac{\pi}{2}$ d. h. längs der Erzeugenden $D S, D S^{\prime}$ und $G_{\infty}$ ist stets $k=0$ (mit Ausnahme der Doppelpunkte $S$ und $S^{\prime}$ ).

Für $u=\frac{p}{\cos v}$, längs der Doppelgeraden $Z$, wird $k=-\frac{\cos ^{2} v}{p^{2} \operatorname{tg}^{2} v}$, dies ist der Maximalwert von $k$ längs einer bestimmten Erzeugenden $v$. 
In den Doppelpunkten $S(v=0)$ und $S^{\prime}(v=\pi)$ auf $Z$ ist $k=-\infty$ and nimmt von hier aus nach beiden Seiten bis zum unendlich fernen Punkt von $Z$ absolut bis zu $k=0$ ab. Auf jeder Erzeugenden nimmt, von ihrem Schnittpunkt mit $Z$ aus, nach beiden Seiten $k$ ebenfalls absolut ab bis zu $k=0$ im Unendlichen.

Mittlere Krümmung:

$$
\begin{gathered}
H=\frac{1}{\rho_{1}}+\frac{1}{\rho_{2}}=\frac{E D^{\prime \prime}-2 F D^{\prime}+G D}{\delta^{2}} \\
=\frac{2 \cos v}{\delta^{3}}\left[\operatorname{tg}^{2} v(p-u \cos v)^{2}-u^{2}+u p \cos v\right] .
\end{gathered}
$$

Die Gleichung zur Bestimmung der Hauptkrüm mungsradien:

$$
\left(D D^{\prime \prime}-D^{\prime 2}\right) \rho^{2}-\left(E D^{\prime \prime}+G D-2 F D^{\prime}\right) \rho+8^{2}=0
$$

wird :

$$
\begin{aligned}
& p^{2} \sin ^{2} v \cdot p^{2}+2 \rho \cos v \sqrt{p^{2} \operatorname{tg}^{2} v+2(p-u \cos v)^{2}} . \\
& .\left\{u \cos v(p-u \cos v)+p \operatorname{tg}^{2} v(p-2 u \cos v)\right\} \\
& \quad-\left[p^{2} \operatorname{tg}^{2} v+2(p-u \cos v)^{2}\right]^{2}=0 .
\end{aligned}
$$

Es wird $\rho_{1}=-\rho_{2}$ und die mittlere Krümmung $H=0$, wenn der Coefficient von $\rho$ verschwindet; dies geschieht zunächst, wenn $v= \pm \frac{\pi}{2}$, also in den Punkten von $G_{\infty}$, wo $p_{1}=-+\infty$ und $\rho_{2}=-\infty$ ist. Den andern Fall werden wir bei den Asymptotenlinien behandeln.

In den Punkten der Leitlinie $L(u=0)$ werden die beiden Hauptkrümmungsradien :

$$
\left.\begin{array}{l}
\rho_{1} \\
\rho_{2}
\end{array}\right\}=\frac{-p}{\sin v \cos v}(\sin v \mp \sqrt{2}) \sqrt{2+\operatorname{tg}^{2} v} .
$$

Im Punkte $D$ derselben, $v=0$ und $\pi$, werden beide unendlich gro $\beta_{*}$ Den beiden Parameterwerten $v$ und $\pi+v$ in irgend einem Punkt der Doppelgeraden $L$ entsprechen zwei Paare von Krümmungsradien, die sich aber nur durch das Vorzeichen unterscheiden.

In den Punkten der Leitlinie $Z\left(u=\frac{p}{\cos v}\right)$ wird:

$$
\left.\begin{array}{l}
P_{1} \\
P_{2}
\end{array}\right\}=\frac{p \operatorname{tg} v}{\cos v}(1+\sqrt{2})
$$

In den Doppelpunkten $S(v=0)$ und $S^{\prime}(v=\pi)$ werden beide Hauptkrümmungsradien zu Null. 
In den Punkten der in der $(y z)$ Ebene liegenden Erzeugenden $D S(v=0)$ und $D S^{\prime}(v=\pi)$ wird der eine Hauptkrümmungsradius $p_{1}$ stets unendlich, und der andere $p_{2}=\sqrt{2} \frac{(p-u)^{2}}{u} ;$ Ausnahme findet nur in den Punkten $S$ und $S^{\prime}$ selbst statt, wo $\rho_{1}=\rho_{2}=0$.

Die Differentialgleichung der Krümmungslinien der Conchoidenfläche wird:

$$
\begin{aligned}
& 2 p \sin v \cos ^{3} v d u^{2}+2 u \cos ^{4} v\left(p-u \cos v-2 p \operatorname{tg}^{2} v\right) \\
& . d u d v-p^{2} \operatorname{tg} v\left(p-2 u \cos v+u \cos ^{3} v\right) d v^{9}=0
\end{aligned}
$$

Die Gleichung ist erfüllt für $v=0$ und $v=\pi$, die zwei Erzeugenden $S D$ und $S^{\prime} D$ sind also Krümmungslinien der Fläche. Die allgemeine Integration lässt sich nicht ausführen.

Die erste Fundamentalform oder das Linienelement a uf der Fläche wird durch den Ausdruck gegeben:

$$
\begin{gathered}
d s^{2}=E d u^{2}+2 F d u d v+G d v^{2}=2 d u^{2}- \\
-2 p \frac{\operatorname{tg} v}{\cos v} d u d v+\left(p^{2}-2 p u \cos ^{3} v+u^{2} \cos ^{1} v\right) \frac{d^{2}}{\cos ^{4} v} .
\end{gathered}
$$

Den Winkel $w$, unter welchem die Parametercurven $u=$ const. (Conchoiden) und $v=$ const. (Erzeugende) sich schneiden erhält man aus:

$$
\cos w=\frac{F}{\sqrt{E \cdot G}}=\frac{p \sin v}{\sqrt{2\left(p^{2}-2 p u \cos ^{3} v+u^{2} \cos ^{4} v\right)}} .
$$

Den Winkel $\alpha$, den eine Erzeugende $v$ mit der Leitlinie $L(u=0)$ bildet, ergibt sich hiernach aus $\cos \alpha=\frac{1}{\sqrt{2}} \sin v$.

Für $v=0$ und $v=\pi$ wird $\alpha=90^{\circ}$ : Erzeugende $S D$ und $S^{\prime} D$ in $(y z)$ Ebene.

Für $v= \pm \frac{\pi}{2}$ wird $\alpha= \pm 45^{\circ}$ : Doppelgerade $G_{\infty}$.

Die Neigung der Erzeugenden gegen $L$ nimmt also von der senkrechten Lage von $D$ aus nach beiden Seiten auf $L$ bis ins Unendliche bis auf $45^{\circ} \mathrm{ab}$, welches der Minimalwert ist. Die Doppelerzeugende $G_{\infty}$ schneidet die beiden Leitlinien $L$ und $Z$ im Unendlichen unter dem Winkel $45^{\circ}$.

Für $u=\infty$ wird $v=90^{\circ}$, d. h. die Erzeugenden der Fläche schneiden ihren unendlich fernen Kreis rechtwinklig.

\section{Tangentialebenen der Fläche.}

Die Gleichung der Tangentialebene in einem Punkte $u, v$ der Fläche in ihrer einfachsten Form lautet: 


$$
\begin{gathered}
x u \sin v \cos ^{2} v-y\left(p-u \cos ^{3} v\right)-z(p-u \cos v) \cos v+ \\
+p(p-u \cos v)=0 .
\end{gathered}
$$

Sie geht durch diejenige Erzeugende $v$ der Fläche, aut welcher der Punkt liegt. Bei constantem $v$ und variablem $u$ stellt die Gleichung das Büschel der Tangentialebenen in sämmtlichen Punkten einer Erzeugenden $v$ dar. Die Tangentialebenenbüschel $S_{1}=0$ und $S_{2}=0$, zweier Erzengenden $v_{1}$ und $v_{z}$ sind projectivisch, es entsprechen sich je zwei solche Ebenen beider Büschel, deren Berührungspunkte auf derselben Conchoide $u$ liegen. Das Erzeugnis der beiden projectivischen Büschel ist ein Hyperboloid, welchem die Geraden $v_{1}$ und $v_{2}$ als Erzengende angehören. Jedes dieser Hyperboloide schneidet die $(x y)$ Ebene in der Leitlinie $L$ und einer zweiten Geraden.

In den Punkten der Doppelerzeugenden $G_{\infty}$ ist $v= \pm \frac{\pi}{2}$, für diese Werte reduciert sich die Gleichung (17) der Tangentialebene auf $y=p$, sie ist unabhängig von $u, d$. h. die Fläche besitzt in allen Punkten von $G_{\infty}$ dieselbe Tangentialebene, es ist die A symptotenebene, $y=p$, der Fläche.

Die Parameter der Erzengenden $D S$ und $D S^{\prime}$ sind $v=0$ und $v=\pi$, für diese wird die Gleichung der Tangentialebene $y \pm z=p$, sie ist unabhängig von $u$, d. h, längs der beiden Erzeugenden $D S$ und $D S^{\prime}$ besitzt die Fläche je eine einzige Tangentialebene, welche auf der $(y z)$ Ebene senkrecht steht. Jede dieser Ebenen schneidet die Fläche außer in der doppelt zu zählenden Erzeugenden $D S$ bezüglich $D S^{\prime}$ noch in der Leitlinie $L$.

Das Büschel der Tangentialebenen durch die Leitlinie Z $\left(u=\frac{p}{\cos v}\right)$ hat die Gleichung:

$$
x=y \operatorname{tang} v
$$

wo $v$ der Winkel ist, den die Tangentialebene mit der positiven $(y z)$ Ebene bildet. In jedem Punkte $z=u==\frac{p}{\cos v}$ von $Z$ gibt es zwei, symmetrisch zur $(y z)$ Ebene liegende Tangentialebenen, entsprechend den zwei Parameterwerten, $\pm v$ des Punktes und jedes Paar gehört zu zwei Punkten von $Z$, die in gleichem Abstande von $O$ liegen, wo der zweite Punkt die Parameter $\pm(\pi+v)$ hat. Jede Tangentialebene durch $Z$ enthält daher zwei Erzeugende, $v$ und $\pi+v$, oder $-v$ und $-(\pi+v)$, die sich auf $L$ schneiden. In den uniplanaren Doppelpunkten $S(u=p, v=0)$ und $S^{\prime}(u=-p, v=\pi)$ fallen die zwei Tangentialebenen in der ( $y z)$ Ebene, im unendlich fernen Punkte von $Z,\left(u=\infty, v= \pm \frac{\pi}{2}\right)$, in der $(x z)$ Ebene zusammen. In den Punkten zwischen $S$ und $S^{\prime}$ sind die Tangentialebenen imaginär. 
Die Paare von Tangentialebenen in den auf einander folgen den Punkten von $Z$ bilden eine Involution von Ebenen, deren reelle Doppelebenen, die $(y z)$ und die $(x z)$ Ebene auf einander senkrecht stehen, die Involution ist also gleichseitig hyperbolise h.

Das Büschel der Tangentialebenen in den Punkten der Leitlinie $L(u=0)$ hat die Gleichung:

$$
y+2 \cos v=p \text {. }
$$

$\mathrm{Zu}$ jedem Punkte von $L$ gehören zwei symmetrisch zur $(y z)$ Ebene liegende, den Parameterwerten $v$ und $\pi+v$ entsprechende Tangentialebenen. Je zwei symmetrisch zum Punkte $D$ liegenden Punkten auf $L$ entspricht dasselbe Tangentialebenenpaar. Jede dieser Ebenen enthält dáher zwei Erzeugende $(t v)$, der Fläche, welche sich auf $Z$ durchschneiden. Im unendlich fernen Punkt, $v= \pm \frac{\pi}{2}$ von $L$ fallen beide Tangentialebenen in der Asymptotenebene $y=p$ zusammen. Im Punkte $D(v=0, \pi)$ stehen sie auf einander senkrecht und sind die Tangentialebenen $y \pm z=p$ längs der Erzeugenden $D S$ und $D S^{\prime}$, sie bilden mit der Asymptotenebene die Winkel $\pm 45^{\circ}$ und sind die äußersten reellen Tangentialebenen durch $L$, bei größerer Neigung werden sie imaginär.

Die Paare der Tangentialebenen durch $L$ bilden ebenfalls eine gleichseitig hyperbolische Involution von Ebenen, deren Doppelebenen die Asymptotenebene $y=z$ und die $(x y)$ Ebene sind, die letztere entspricht den imaginären Parametern $v=\frac{ \pm}{\text { Diese }}$.

Diese beiden gleichseitig hyperbolischen Involutionen der Tangentialebenen, deren Scheitelkanten $L$ und $Z$ der Richtung nach auf einander senkrecht stehen, sind zu einander projecti$v$ isch. Jedem Werte des Parameterwinkels $v$ entspricht in beiden je ein Ebenenpaar und die auf einander folgenden Schnittlinien aller Paare entsprechender Ebenen der beiden Involutionen erzeugen eine Regelfläche 4. Ordnung, unsere Conchoidenfläche. Die Scheitelkanten $Z$ und $L$ sind Doppellinien derselben. Der $(y z)$ Ebene als Doppelebene $(v=0, \pi)$ der Involution $Z$ entsprechen in der Involution $L$ die zwei Ebenen $y \pm z=p$, die Tangentialebenen längs der Erzeugenden $D S$ und $\bar{D} S$, welche in diesen Ebenen doppelt zu zählen sind. Der $(x z)$ Ebene als Doppelebene $\left(v= \pm \frac{\pi}{2}\right)$ der Involution $Z$ entspricht die Asymptotenebene $y=p$ als Doppelebene der Involution $L$, beide sind zu einander parallel, ihre Schnittlinie ist die Doppelerzeugede $G_{\infty}$ der Fläche. Der zweiten Doppelebene der Involution $L$, der $(x y)$ Ebene entsprechen zwei imaginäre Ebenen der Involution $Z$, die Tangentialebenen $y= \pm i x$ im Nullpunkt $O$ auf der isolierten Strecke $S S^{\prime}$ der Doppelgeraden $Z$. 
Verschiebt man die Involution $L$ parallel mit sich selbst, so dass $L$ mit der $x$-Axe zusammenfällt, und die beiden Scheitelkanten $x$ und $Z$ sich schneiden, so erzeugen die beiden Involutionen den Leitkegel der Conchoidenfäche, die Doppelebene $(x z)$ sondert sich ab.

Durch Elimination des Parameters $v$ aus den Gleichungen (18) und (19) der beiden Involutionen ergibt sich die Gleichung der Conchoidenfläche.

Verschwindet in der Gleichung (17) der Tangentialebene der Coefficient ron $y$ :

also

$$
p-u \cos ^{3} v=0
$$

$$
u=\frac{p}{\cos ^{3} v}
$$

so wird dieselbe parallel zur $y$-Axe. Setzt man diesen Wert von $u$ in den Flächengleichungen (8) ein, so erhält man die Gleichungen derjenigen Curve auf der Fläche, in deren Punkten die zur $y$ Axe parallelen Tangentialebenen di e Flä che berühren, nämlich :

$$
x=-p \operatorname{tg}^{3} u ; \quad y=-p \operatorname{tg}^{2} v, \quad z=\frac{p}{\cos ^{3} v} .
$$

Die Coordinate $y$ ist stets negativ. Für $v=0, \pi$ wird $x=$ $=y=0$ und $z= \pm p$, es sind die Doppelpunkte $S^{\prime}$ und $S^{\prime}$ der Fläche, in welchen die Coordinate $z$ den absolut kleinsten Wert hat. Für $v= \pm \frac{\pi}{2}$ wird $x=y=z=\infty$.

Die Projectionen der Curve auf die Coordinatenebenen sind:

$(x y)$ Ebene: $y^{3}=-p x^{2}=\mathrm{Neilsch}$ e Parabel mit Spitze in $O$. (20a) (yz)Ebene: $(p-y)^{3}=p^{2} z^{2}=$ Neilsehe Parabel mit Spitze in $D$. $(x z)$ Ebene: $z^{\frac{2}{3}}-x^{\frac{2}{3}}=p^{\frac{2}{3}}=$ Evolute der gleichseitigen Hy perbel

$z^{2}-x^{2}=\frac{p^{2}}{4}$, welche in der Ebene $y=\frac{p}{2}$ liegt und die Durchschnittslinie des Leitkegels mit der Conchoidenfläche ist. (Gleichung 7). Diese Evolute ist die Enveloppe derjenigen Geraden, in welchen die zur $y$-Axe parallelen Tangentialebenen der Fläche die $(x z)$ Ebene oder eine dazu parallele Ebene $\left(y=\frac{p}{2}\right.$ z. B.) durchschneiden.

Die Raumcurve auf der Fläche selbst hat in $S$ und $S^{\prime}$ Spitzen und geht von diesen aus in zwei congruenten Theilen, von der 
Form räumlicher Neilscher Parabeln, auf der negativen Seite der $(x z)$ Ebene nach oben und nach unten auf den beiden Wulsten der Fläche ins Unendliche.

\section{Centralpunkt und Strictionslinie.}

Legt man durch irgend eine Erzeugende $v$ das Büschel der Tangentialebenen, so können diese paarweise so einander zugeordnet werden, dass je zwei auf einander senkrecht stehen. Alle diese Paare rechtwinkliger Tangentialebenen bilden eine Involution, somit bilden ibre Berührungspunkte auf der Erzeugenden eine Involution von Punkten, in der das Doppelverhältnis von irgend vier Punkten gleich dem der conjugierten Tangentialebenen ist. Das Centrum der Involution der Berührungspunkte auf einer Exzeugenden ist der Berührungspunkt derjenigen Tangentialebene, welche senkrecht steht anf der Tangentialebene im unendlich fernen Punkt dieser Erzeugenden, wir nennen ihn den Centralpunkt der Erzeugenden.

Die Gleichung der Tangentialebene im Punkte $(u, v)$ ist nach $(17)$ :

$$
\begin{gathered}
x u \sin v \cos ^{2} v-y\left(p-u \cos ^{2} v\right)-z(p-u \cos v) \cos v+ \\
+p(p-u \cos v)=0 .
\end{gathered}
$$

Die Tangentialebene im unendlich fernen Punkt $(u=\infty)$ der Erzeugenden $v$ wird:

$$
x \sin v \cos v+y \cos ^{2} v+z \cos v=p .
$$

Soll diese auf der ersteren senkrecht stehen, so muss die Bedingung erfüllt sein :

$$
u \cos v-p=0
$$

hieraus folgt als Parameter des Centralpunktes auf der Erzeugenden $v$ der Wert $u=\frac{p}{\cos v}$ d. h. er liegt auf der $z$-Axe, und die Gleichung der zuhörigen Tangentialebene wird:

$$
x=y \cdot \operatorname{tg} v,
$$

sie geht durch die betreffende Erzeugende und die z-Axe. Wir finden also:

Der Centralpunkt jeder Erzeugenden der Conchoidenfläche liegt in ihrem Schnittpunkt mit der Doppelgeraden, der Leitlinie $Z$, es ist derjenige Punkt der Erzengenden, in welchem sie ron der nächst folgenden Erzeugenden den kürzesten Abstand hat. Der Ort aller dieser Centralpunkte ist die Leitlinie $Z$, sie heißt die Strictionslinie der Fläche.

Längs jeder Erzeugenden nimmt der absolute Betrag des Krümmungsmaßes $K$ im Centralpunkt seinen Maximalwert an, 
$K=\frac{-\cos ^{2} v}{p^{2} \operatorname{tg}^{2} v}$, und nähert sich um so mehr der Null, je weiter sich ein Punkt auf der Erzeugenden beiderseits vom Centralpunkt entfernt. Das Maximum auf den verschiedenen Erzeugenden in den Punkten der Strictionslinie $Z$ ändert seinen Wert continuierlich, es hat seinen absolut größten Wert, $K=-\infty$, in den Doppelpunkten $S(v=0)$ und $S^{\prime}(v=\pi)$, nimmt oberhalb bezüglich unterhalb dieser Punkte stetig ab bis $K=0 \mathrm{im}$ unendlich fernen Punkt der Strictionslinie.

Die Tangentialebenen in den unendlich fernen Punkten der Erzeugenden bilden die asymptotisehe Developpable der Conchoidenfläche, sie ist von der 6. Ordnung, sie schneidet die $(y z)$ und die $(x y)$ Ebene in Geraden, deren Enveloppe die beiden Parabeln $z^{2}=-4 p y$ und $x^{2}=-4 p(y-p)$ sind. Die zu diesen Tangentialebenen parallel durch den Nullpunkt gelegten Ebenen umhüllen den Leitkegel.

\section{Normalen der Fläche.}

Die Gleichungen der Normalen in einem Punkte $(u, v)$ der Conchoidenfläche ergeben sich als:

$$
\begin{aligned}
& x(p-u \cos v)-(p-u \cos v)^{2} \operatorname{tg} v=u \cos v \cdot \sin v(u-z) \text { und } \\
& y(p-u \cos v)-(p-u \cos v)^{2}=\left(u \cos ^{2} v-\frac{p}{\cos v}\right)(u-z) .
\end{aligned}
$$

Eliminiert man aus beiden Gleichungen den Parameter $u$, so ergibt sich als geometrischer Ort aller Normalen längs einer Erzeugenden $v$ die Fläche zweiten Grades:

$$
\begin{gathered}
(y \sin v-x \cos v)(x \sin v+y \cos v-z)= \\
\frac{p}{\cos ^{2} v}(x+z \sin v-p \operatorname{tg} v) .
\end{gathered}
$$

Die Fläche ist eine Regelfläche, sie kann erzeugt werden durch die folgenden zwei Paare projectivischer Ebenenbüschel:

und

$$
\left\{\begin{array}{l}
y \sin v-x \cos v=\frac{\lambda p}{\cos ^{2} v} \\
\lambda(x \sin v+y \cos v-z)=x+z \sin v-p \operatorname{tg} v
\end{array}\right.
$$

$$
\left\{\begin{array}{l}
x \sin v+y \cos v-z=\frac{\lambda p}{\cos ^{2} v} \\
\lambda(x \sin v-y \cos v)=x+2 \sin v-p \operatorname{tg} v
\end{array}\right.
$$

wo $\lambda$ ein veränderlicher Parameter bedeutet. 
Das erste Paar liefert die eine Schar von Erzeugenden, die sämmtlich parallel sind der festen Ebene $y \sin v-x \cos v=0$ oder $x=y \operatorname{tg} v$, welche durch die $z$-Axe und die betreffende Erzeugende geht und welche der parallelen Ebenenschar selbst angehört. Die zweite Schar von Erzeugenden der Fläche 2. Grades ist parallel der festen Ebene $x \sin v+y \cos v-z=0$, welche senkrecht steht auf der zugehörigen Erzetugenden $v$, es ist dies die Normalenschar.

Die Normalenfläche längs jeder Erzeugenden ist also ein hyperbolisches Paraboloid und da die ebenen Schnitte parallel der $(x y)$ Ebene mit der Fläche gleichseitige Hyperbeln sind, so ist das Paraboloid gleichseitig, der Scheitel desselben liegt im Centralpunkt der Erzeugenden, also auf der Leitlinie $Z$.

Die den Erzeugenden $D S(v=0)$ und $D S^{\prime}(v=\pi)$ zugehörigen Normalenparaboloide reducieren sich auf zwei Ebenen, die $(y z)$ Ebene und die darauf senkrechte Tangentialebene längs $D S$ beziuglich $D S^{\prime}$.

Für $u=0$ erhält man die Normalen in den Punkten der Leitlinie $L$ der Conchoidenfläche, nämlich:

$$
x=p \operatorname{tg} v \text { and } y \cos v-z=p \cos v .
$$

In jedem Punkt von $L$ gibt es zwei Normalen, symmetrisch zur Asymptotenebene $y=p$, entsprechend den Werten $v$ und $180^{\circ}+v$, sie stehen auf $L$ senkrecht. Die beiden Normalen in $D(v=0, \pi)$ stehen auf einander senkrecht und fallen mit $D S^{\prime}$ und $D S$ zusammen, sie bilden mit der Asymptotenebene die Winkel $\pm 45^{\circ}$. Vom Punkte $D$ aus nach beiden Seiten auf $L$ nähern sich die Normalen in ihrer Neigung immer mehr der $(x y)$ Ebene und im unendlich fernen Punkt von $L$ fallen beide in der $(x y)$ Ebene zusammen.

Durch Elimination von $v$ aus den obigen zwei Normalengleichungen ergibt sich die Normalenfläche längs der Leitlinie $L$, nämlich:

$$
p^{2}(y-p)^{2}=z^{2}\left(x^{2}+p^{2}\right) .
$$

Macht man die Leitlinie $L$ zur $x$-Axe, so wird ihre Gleichung:

$$
z^{\prime 2}\left(x^{\prime 2}+p^{2}\right)=y^{2} y^{\prime 2} \text {. }
$$

Sie ist eine Regelfläche 4 . Ordnung, $L$ ist Doppellinie and die unendlich ferne Gerade der $(x y)$ Ebene enthält zwei zusammenfallende Erzeugende derselben. Eine Ebene $z= \pm c$, parallel zur $(x y)$ Ebene schneidet diese Fläche in der Hyperbel:

$$
\frac{y^{\prime 2}}{c^{2}}-\frac{x^{\prime 2}}{p^{2}}=1
$$

ihre imaginäre Axe ist constant $=p$. Für $z=c=0$ reduciert sie sich auf die Leitlinie $L$ und für $z=c= \pm p$ wird sie gleich- 
seitig, der eine Scheitel liegt in $S$ bezüglich $S^{\prime}$. Diese Normalenfläche hat mit der Conchoidenfläche die Leitlinie $L$ und die Geraden $S D$ und $S^{\prime} D$ gemein.

Die Gleichung der Normalen in einem Punkte $u=\frac{p}{\cos v}$ der Leitlinie $Z$ sind:

$$
z=\frac{p}{\cos v} \text { and } x=-\operatorname{cotg} v \cdot y \text {. }
$$

In jedem Punkte von $Z$ gibt es zwei solche Normalen, $v$ und $2 \pi-v$, die senkrecht auf $Z$ stehen und symmetrisch zur $(x z)$ Ebene liegen. Für $v=0$ und $2 \pi$, im Doppelpunkte $S$ fallen sie zusammen in einer zur $x$-Axe parallelen Geraden $(z=p, y=0)$, ebenso im Punkte $S^{\prime}$ für $v= \pm \pi$. Im unendlich fernen Punkt von $Z\left(v= \pm \frac{\pi}{2}\right)$ fallen die beiden Normalen mit der unendlich fernen Geraden der $(y z)$ Ebene zusammen, parallel zur $y$-Axe.

Die Normalen längs der Leitlinie $Z$ bilden ebenfalls eine Regelfläche 4. Ordnung, für welche $Z$ Doppellinie ist, auf der alle Erzeugenden senkrecht stehen; ihre Gleichung ist:

$$
x^{2} z^{2}=p^{2}\left(x^{2}+y^{2}\right) \text {. }
$$

Die zur $(y z)$ Ebene parallelen ebenen Schnitte, $x=a$, sind Hyperbeln $\frac{z^{2}}{p^{2}}-\frac{y^{2}}{a^{2}}=1$, die zar $(x, y)$ Ebenen parallen Schnitte sind Curven 4. Ordnung.

Die in der Ebene $z= \pm c$ liegenden Normalen der Fläche haben die Gleichung:

$$
p y= \pm x \sqrt{c^{2}-p^{2}}
$$

sie sind nur reell, wenn $c$ absolut $\overline{>} p$ ist; für $c= \pm p, \mathrm{~d}$. h. in $S$ und $S^{\prime}$ fallen sie in $y=0$ zusammen, für $c= \pm p \sqrt{2}$ stehen sie auf einander senkrecht und für $z=c= \pm \infty$ fallen sie in $x=0, z=-\infty$ zusammen. Die Fläche besteht aus zwei congruenten getrennten Theilen mit je einer scharfen Schneide parallel zur $x$-Axe durch $S$ und $S^{\prime}$ and einer dritten solchen Schneide in der unendlich fernen Geraden der $(y z)$ Ebene, parallel der $y$-Axe.

Diese Normalenfläche schneidet die Conchoidenfläche, außer in $Z$, in einer Curve, deren Projection auf die $(z y)$ Ebene die gleichseitige Hyperbel $(y-p)^{2}-z^{2}=p^{2}$ ist, ihre Halbaxe ist $p$, Mittelpunkt $D$. Die Projektion auf die $(x y)$ Ebene besteht auf zwei congruenten gleichseitigen Hyperbeln $x y-p(x \pm y)=0$, sie schneiden sich in $O$, Halbaxe $=p \sqrt{2}$, Mittelpunkte $M_{1}(-p, p)$ und $M_{2}(p, p)$, die Leitlinie $L$ ist gemeinschaftliche Asymptote, die zwei anderen Asymptoten sind $x= \pm p$. Die Projection auf die $(x z)$ Ebene ist die Curve 4. Ordnung $(x-p)^{2}\left(z^{2}-p^{2}\right)=p^{4}$. 


\section{Asymptotenlinien oder Haupttangentencurven.}

Die Erzeugenden einer Regelfläche bilden die eine Schar vor Asymptotenlinien. Die Differentialgleichung der zweiten Schar, die sogenannte $z$ weite Fundamentalform der Fläche:

$$
D d u^{2}+2 D^{\prime} d u \cdot d v+D^{\prime \prime} d v^{2}=0 \text {. }
$$

(Bianchi, Differentialgeometrie p. 87) reduciert sich, da $D=0$ ist, auf

$$
2 D^{\prime} d u+D^{\prime \prime} d v=0 \text {. }
$$

Für die Conchoidenfläche wird diese Gleichung:

$$
2 p \operatorname{tg} v \cdot d u+u\left(p-u \cos v-2 p \operatorname{tg}^{2} v\right) d v=0
$$

oder

$$
\frac{d u}{d v}-\frac{\cos v}{2 p \operatorname{tg} v} \cdot u^{2}+\left(\frac{1-2 \operatorname{tg}^{2} v}{2 \operatorname{tg} v}\right) u=0 .
$$

Es ist dies die Form der Riccati'schen Difterentialgleichung. Um sie zu integrieren, substituiert man $u=\theta t$, und bestimmt $\Theta$ so, dass

wird, also

$$
\frac{d \Theta}{d v}+\frac{1-2 \operatorname{tg}^{2} v}{2 \operatorname{tg} v} \Theta=0
$$

$$
\frac{d \Theta}{\Theta}=\frac{2 \operatorname{tg}^{2} v-1}{2 \operatorname{tg} v} d v=\left(\operatorname{tg} v-\frac{1}{2} \operatorname{cotg} v\right) d v .
$$

Hieraus $\log \theta=-\log \cos v-\frac{1}{2} \log \sin v=\log \frac{1}{\cos v \sqrt{\sin v}}$

$$
\Theta=\frac{1}{\cos v \sqrt{\sin v}} .
$$

Die Differentialgleichung geht nun über in:

also

$$
\frac{d t}{t v}=\frac{\cos v}{2 p \operatorname{tg} v} \cdot \theta t^{2}=\frac{t^{2}}{2 p \operatorname{tg} v \sqrt{\sin v}},
$$

$$
\frac{d t}{t^{2}}=\frac{d v}{2 p \operatorname{tg} v \sqrt{\sin v}}=\frac{1}{2 p(\sin v)^{\frac{3}{2}}}=\frac{\cos v d v}{2 p(\sin v)^{\frac{3}{2}}},
$$

integriert:

$$
\frac{1}{t}=\frac{1}{p \sqrt{\sin v}}+C
$$


Weil nun $\frac{1}{t}=\frac{\theta}{u}=\frac{1}{u \cos v \sqrt{\sin v}}$ ist, so wird das Integral der Differentialgleichung (26) oder die Gleichung der zweiten Schar von Asymptotenlinien:

oder

$$
(p-u \cos v)^{2}=C^{2} p^{2} u^{2} \cos ^{2} v \sin v,
$$

$$
u=\frac{p}{\cos v\left(1 \pm C_{p} \sqrt{\sin v)}\right.}
$$

wo $C$ eine beliebige Constante bedeutet. Setzt man diesen Wert von $u$ in den Flächengleichungen ein, so erhält man die Coordinaten der Punkte einer Asymptotenlinie $C$ in der Form:

$$
\begin{aligned}
& x=\frac{ \pm C p^{2} \sqrt{\sin v}}{1 \pm C p \sqrt{\sin v}} \cdot \operatorname{tang} v \\
& y=\frac{ \pm C p^{2} \sqrt{\sin v}}{1 \pm C p \sqrt{\sin v}} \\
& z=\frac{p}{\cos v(1 \pm C p \sqrt{\sin v)}}
\end{aligned}
$$

Jedem Werte des veränderlichen Parameters $C$ entspricht eine bestimmte Asymptotenlinie, welche, da $z$ nie gleich Null werden kann, aus zwei congruenten Ästen oberbalb und unterhalb der $(x y)$ Ebene besteht. Negativen Werten von $C$ entsprechen dieselben Curven wie positiven Werten. Für $v=0$ und $\pi$ wird $x=y=0, z= \pm p$, unahbängig von $C, \mathrm{~d}$. h. Alle A s ymptotenlinien gehen dureh die Doppelpunkte $S$ und $S^{\prime}$ der Fläche.

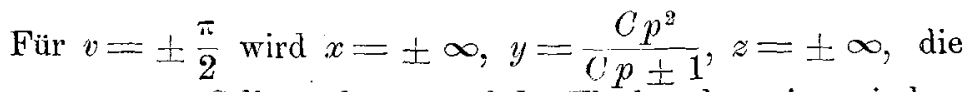
Asymptotencurve $C$ liegt also so auf der Fläche, dass sie zwischen den zwei parallelen Ebenen $y=\frac{C p^{2}}{C p+1}$, welche gewissermaben ihre Asymptotenebenen sind, ins Unendliche verläuft. Für $C=0$ fallen beide Ëbenen mit der $(x z)$ Ebene, für $C=\infty$ mit der Asymptotenebene $y=p$ der Fläche zusammen.

Die Projection einer Asymptotenlinie $C$ hat die Gleichung:

In der $(x y)$ Ebene:

$x^{2}\left\{C^{4} p^{4}(p-y)^{4}-y^{4}\right\}=y^{6}$, Asymptoten $y=\frac{C p^{2}}{C p \pm 1}$.

In $\operatorname{der}(y z)$ Ebene:

$z^{2}\left\{C^{4} p^{4}(p-y)^{4}-y^{4}\right\}=C^{4} p^{4}(p-y)^{6}$, ssymptoten $y=\frac{C p^{2}}{C \pm 1}$. 
In $\operatorname{der}(x z)$ Ebene ist sie ebenfalls vom sechsten Grade. Die Asymptotenlinien sind also Ra u curven sechster Ordnung.

Die Asymptotenlinie $C=0$ besteht aus der 4 fachen Leitlinie $Z$ und der Doppelgeraden $G_{\infty}$.

Die Asymptotenlinie $C=\infty$ besteht aus der 4fachen Leitlinie $L$ und den zwei Erzeugenden $D S$ und $D S^{\prime}$.

Die Curven auf der Fläche, in deren Punkten sich die Asymptotenlinien der beiden Scharen rechtwinklig durchschneiden, d. h. der Ort der Punkte, in welchen die obigen Asymptotenlinien (28) die Erzeugenden der Conchoidenfläche rechtwinklig schneiden, ist zugleich der Ort der Punkte, in welchen die mittlere Krümmung $H=0$ ist. Die Gleichung dieser Flächencurve ist daher nach Gleichung (13):

$$
(p-u \cos v)^{2} \operatorname{tg}^{2} v-u^{2}-p(p-u \cos v)+p^{2}=0 .
$$

Führt man mit Hilfe der Flächengleichungen:

$$
(p-u \cos v) \operatorname{tg} v=x, \quad p-u \cos v=y, \quad u=z
$$

rechtwinklige Coordinaten ein, so wird die Gleichung:

$$
x^{2}-z^{2}-p y+p^{2}=0 .
$$

Die gesuchte Ortscurve ist die Durchschnittslinie dieses gleichseitig hyperbolischen Paraboloids mit der Conchoidenfläche. Die Projection der Schnittcurve auf die $(x y)$ Ebene hat die Gleichung:

$$
(p-y) y^{3}+p x^{2}(2 y-p)=0 .
$$

Der Nullpunkt ist Spitze, $y=\frac{p}{2}$ Asymptote.

Diejenige auf die $(y z)$ Ebene: $z^{2}\left(p^{2}-2 p y\right)+(y-p)^{2}\left(y^{2}+p y-p^{2}\right)=0$, Asymptote $y=\frac{p}{2}$.

Die Raumcurve selbst ist von der 8. Ordnung, sie besitzt in $D$ einen Doppelpunkt und geht durch $S$ und $S^{\prime}$.

\section{Orthogonale Trajectorien der Erzeugenden und geodätische Linien.}

Zwei Curvenscharen $\varphi(u, v)=c$ und $\downarrow(u, v)=C$ auf einer Fläche durchschneiden sich orthogonal, wenn der gemischte Differentialparameter der beiden F'unctionen $\nabla(\varphi, \varphi)=0$ ist. Die Differentialgleichung der zu einer gegebenen Curvenschar $\varphi(u, v)=C$, orthogonalen Trajectorien ist (Bianchi p. 66):

$$
\left(E \frac{\partial \varphi}{\partial v}-F \frac{\partial \varphi}{\partial u}\right) d u+\left(F \frac{\partial \varphi}{\partial v}-G \frac{\partial \varphi}{\partial u}\right) d v=0 .
$$


Die Gleichung der Schar der Erzeugenden der Fläche ist nun $\varphi=v=c=$ const. Die Differentialgleichung ihrer orthogonalen Trajectorien reduciert sich auf

$$
E d u+F d v=0
$$

also

$$
d u-\frac{p}{2} \frac{\sin v}{\cos ^{2} v} d v=0 \text { oder } d u+\frac{p}{2} \frac{d \cos v}{\cos ^{2} v}=0,
$$

integriert :

$$
u-\frac{p}{2} \cdot \frac{1}{\cos v}=C
$$

also

$$
u=C+\frac{p}{2} \frac{p}{\cos v}
$$

Dies ist die Parametergleichung der orthogonalen Trajectorien der Erzeugenden der Fläche; setzt man diesen Wert von $u$ in den Flächengleichungen ein, so erhält man die Gleichungen der Trajectorien selbst:

(31) $x=\left(\frac{p}{2}-C \cos v\right) \operatorname{tg} v, \quad y=\frac{p}{2}-C \cos v, \quad z=C+\frac{p}{2 \cos v}$.

Jedem positiven oder negativen Werte von $C$ entspricht eine bestimmte Trajectorie; für $C=0$ werden ihre Gleichungen:

$$
x=\frac{p}{2} \operatorname{tg} v, \quad y=\frac{p}{2}=\text { const. }, \quad z=\frac{p}{2 \cos v} .
$$

Durch Elimination von $v$ folgt: $z^{2}-x^{2}=\frac{p^{2}}{4}$, d. h.:

Die zu den Erzeugenden der Conchoidenfläche orthogonale Trajectorie $C=0$ ist eine gleichseitige Hyperbel von der Halbaxe $\frac{p}{2}$, welche in der zur Asymptotenebene parallelen Ebene $y=\frac{p}{2}$ liegt, sie ist identisch mit derjenigen Hyperbel (7), in welcher der Leitkegel die Fläche schneidet. Diese Hyperbel durchschneidet also. alle Erzeugenden rechtwinklig.

Die Trajectorie $C==\infty$ liegt ganz im Unendlichen, sie besteht aus dem in der Ebene $z=\infty$ liegenden unendlich großen Kreis und der Doppelgeraden $G_{\infty}$. Hieraus folgt:

Alle Erzeugenden der Fläche schneiden den unendlich fernen Kreis derselben rechtwinklig, wie auch aus der Beschaffenheit des Leitkegels hervorgeht.

Durch Elimination der Veränderlichen $v$ aus je 2 der Gleichungen (31) erhält man die Projectionen der orthogonalen Tra- 
jectorien auf die drei Coordinatenebenen. Diejenige auf die $(x y)$ Ebene wird:

$$
\left(y-\frac{p}{2}\right)^{2}\left(x^{2}+y^{2}\right)=C^{2} y^{2}
$$

Diese Projectionen sind Conchoiden vom Parameter $s=C$, dem Nullpunkt als Pol und der gemeinschaftlichen Leitlinie $y=\frac{p}{2}$ und zwar erhält man: Punkt $O$.

Füur $C=0$, die doppelte Leitlinie $y=\frac{p}{2}$ und den conjugierten

Für $C$ absolut $<\frac{p}{2}$, eine Conchoide mit zwei Ästen und dem conjugierten Punkt $O$.

Für $C= \pm \frac{p}{2}$, eine Conchoide mit Spitze in $O$.

Für $C$ absolut $>\frac{p}{2}$, eine Conchoide mit Doppelpunkt in $O$.

Für $C=\infty$, unendlich großer Kreis um $O$ und doppelte $x$-Axe.

Die Projectionen der Trajectorien auf die $(y z)$ Ebene bilden ein System gleichseitiger Hyperbeln von der Gleichung:

$$
\left(y-\frac{p}{2}\right)(z-C)+\frac{p}{2} c=0
$$

Asymptoten : $y=\frac{p}{2}=$ const. und $z=C$,

$$
\text { Mittelpunkt: }\left(y=\frac{p}{2}=\text { const., } z=C\right) \text {. }
$$

Alle diese Hyperbeln gehen durch den Punkt $D(x=0$, $y=p, z=0$ ) auf $L$.

Für $C=0$ zerfällt die Hyperbel in die beiden Geraden $y=\frac{p}{2}$ und $z=0$.

Die Projectionen auf die $(x z)$ Ebene sind Curven 4. Ordnung:

$$
4 x^{2}(z-C)^{2}=(z-2 C)^{2}\left\{4(z-C)^{2}-p^{2}\right\} .
$$

Die Gerade $z=C$ ist Asymptote der Curve mit imaginären Berührungspunkten; $x$ wird nur reell, wenn $z>C+\frac{p}{2}$ oder $<C-\frac{p}{2}$ ist, die Geraden $z=C \pm \frac{p}{2}$ sind Tangenten in den Scheiteln der Curve auf der $z$-Axe. Die Curve besteht aus zwei unendlichen Ästen oberhalb bezüglich unterhalb dieser beiden Tangenten, der obere Ast besitzt eine Schleife mit Doppelpunkt 
in $z=2 C$ auf der $\approx-A x e$, insofern $C$ absolut $>\frac{p}{2}$ ist. Für $C=\frac{p}{2}$ wird er zur Spitze in $S(z=p)$ mit der $z$-Axe als Rückkehrtangente.

Für $C=0$ zerfällt die Curve in $z^{2}=0$, die doppelte $x$-Axe und in die gleichseitige Hyperbel $z^{2}-x^{2}=\frac{p^{2}}{4}$.

Aus den Gleichungen (31) ergeben sich die Coordinaten der Schnittpunkte der orthogonalen Trajectorie $C$ mit der $(x y)$ Ebene für $\cos v=\frac{-p}{2 C}$, nämlich :

$$
x= \pm \sqrt{4 C^{2}-p^{2}} \text { und } y=p,
$$

diese zwei Schnittpunkte auf $L$ liegen symmetrisch zum Punkte $D(0, p)$, sie sind nur reell, wenn $C$ absolut $>\frac{p}{2}$ ist, für $C= \pm \frac{p}{2}$ fallen sie in $D$ zusammen, die Trajectorie berührt hier die Leitlinie $L$. Zwei Trajectorien, die zwei gleichen, aber entgegengesetzten Werten von $C$ entsprechen, durchschneiden sich in zwei Punkten auf $L$. Aus den Coordinaten der Schnittpunkte folgt:

$$
x^{2}+y^{2}=4 C^{2},
$$

d. h. die Constante $2 C$ ist gleich dem Radius vector jedes der beiden Schnittpunkte der Trajectorie mit der $(x y)$ Ebene.

Die Schnittpunkte der Trajectorie mit der $(y$ ə) Ebene erhält man für $v=0$ und $\pi$, nämlich: $y=\frac{p}{2} \mp C, z=C \pm \frac{p}{2}$. Beide Schnittpunkte liegen auf der Erzeugenden $D S$ bezüglich $D S^{\prime}$ and sind für jedes $C$ reell; für $C=\frac{p}{2}$ fällt der eine Schnittpunkt in $S(y=0, z=p)$, der zweite in $D(y=p, z=0)$.

Aufer für $v=0$ und $\pi$ wird noch $x=0$ für $\cos v=\frac{p}{2} c$, wobei zugleich auch $y=0$ und $z=2 C$ wird. Dieser Punkt der Trajectorie auf der $z$-Axe ist Doppelpunkt, Spitze (in $S$ bez. $S^{\prime \prime}$ ) oder conjugierter Pankt, je nachdem $C$ absolut $\frac{p}{2}$ ist.

Aufer in diesem reellen oder imaginären Doppelpunkt wird die $(x z)$ Ebene in keinem andern Punkt geschnitten.

Aus diesen Untersuchungen folgt, dass die orthogonalen Trajectorien der Erzeugenden der Conchoidenfläche Raumeurven 4. Ordnung sind. Solange $C$ absolut $<\frac{p}{2}$ ist, besteht die Curve aus zwei unendlichen einfachen Zweigen, die zwischen der $(x z)$ Ebene und der Asymptotenebene $y=p$ verlaufen, für ein positives $C$ oberhalb, für ein negatives $C$ unterhalb der $(x y)$ Ebene. Ist 
$C$ absolut $>\frac{p}{2}$, so besitzt die Trajectorie einen Doppelpunkt auf der Leitlinie $Z$ und schneidet die $(x y)$ Ebene in zwei Punkten auf $L$. Die Trajectorien $C= \pm \frac{p}{2}$ bilden den Übergang, ein Ast besitzt, in $S$ bezüglich $S^{\prime}$ eine Spitze, während der zweite Ast die Leitlinie $L$ in $D$ oberhalb, bez. unterhalb der $(x y)$ Ebene berührt.

Weil die Parametercurven $v=$ const. als Erzeugende eine einfach unendliche Schar geodütischer Linien auf der Conchoidenfläche sind, so sind ihre orthogonalen Trajectorien sogenannte geodätisch parallele Linien. Die Strecken, welche auf den Erzeugenden $v$ von irgend zwei ihrer orthogonalen Trajectorien ausgeschnitten werden, sind alle gleich lang. Beide Curvensysteme bilden ein geodätisches Orthogonalsystem. Nimmt man. diese beiden Systeme, die Erzeugenden $v$ und ihre orthogonalen Trajectorien $C$, als Parametercurven der Conchoidenfläche, so werden ihre Gleichungen nach (31), wenn man gleichzeitig das veränderliche $C$ durch $\frac{u}{\sqrt{2}}=$ ersetzt:

$$
\begin{aligned}
& x=\left(\frac{p}{2}-\frac{u}{\sqrt{2}} \cos v\right) \operatorname{tg} v \\
& y=\left(\frac{p}{2}-\frac{u}{\sqrt{2}} \cos v\right) \\
& z=\frac{u}{\sqrt{2}}+\frac{p}{2 \cos v}
\end{aligned}
$$

$u$ und $v$ heipen die geodätischen Coordinaten eines Flächenpunktes. Die Fundamentalgrößen erster Ordnung werden:

$$
E=1, \quad F=0, \quad G=\frac{(\sqrt{2} \cdot u \cos v-p)^{2}+2 p^{2} \operatorname{tg}^{2} v}{4 \cos ^{2} v}
$$

und das Quadrat des Linienelementes auf der Fläche wird:

$$
d s^{2}=d u^{2}+G \cdot d v^{2} \text {. }
$$

Die allgemeine Differentialgleichung der geodätischen Linien nimmt nun die einfachere Gestalt an:

$$
2 G \frac{d^{2} u}{d v^{2}}-2 \frac{\partial G}{\partial u} \cdot\left(\frac{d u}{d v}\right)^{2}-\frac{\partial G}{\partial v} \cdot \frac{d u}{d v}-G \frac{\partial G}{\partial u}=0 .
$$

Dabei hat $G$ den obigen Wert und

$$
\frac{\partial G}{\partial u}=\frac{u \sqrt{2} \cos v-p}{\sqrt{2} \cos v}
$$




$$
\frac{\partial G}{\partial v}=\frac{p \operatorname{tg} v}{2 \cos ^{4} v}\left\{2 p\left(1+\sin ^{2} v\right)-\cos ^{2} v(u \sqrt{2} \cos v-p)\right\}
$$

Die Integration dieser Gleichung lässt sich aber nicht ausführen.

Eine geodätische Linie ist bestimmt durch einen Flächenpunkt $P(u, v)$ und die Richtung $\frac{d v}{d u}$, in welcher sie durch denselben hindurchgeht. Bedeutet $\theta$ den Neigungswinkel, den die geodätische Linie mit den Parametercurven $v$ bildet, so kann nach Gauss die Differentialgleichung der geodätischen Linien folgenderweise zerlegt werden:

$$
\begin{gathered}
\cos \vartheta=\frac{1}{\sqrt{E^{\prime}}}\left(E \cdot \frac{d u}{d s}+F \cdot \frac{d v}{d s}\right) \\
\text { und } d \vartheta=\frac{1}{\sqrt{G}} \frac{\partial \sqrt{E}}{\partial v} \cdot d u-\frac{1}{\sqrt{E}} \frac{\partial \sqrt{G}}{\partial u} d v .
\end{gathered}
$$

Legen wir nun obiges geodätisches Orthogonalsystem zu Grunde, so reducieren sich diese Gleichungen zu:

$$
\cos \theta=\frac{1}{\sqrt{1+G\left(\frac{d v}{d u}\right)^{2}}}
$$

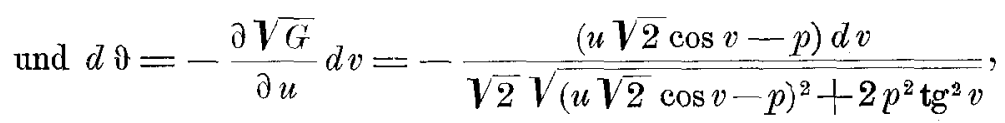
wo $G$ den obigen Wert hat. Die Integration der zweiten Gleichung führt auf ein elliptisches Integral.

\section{Orthogonale Trajectorien der Conchoiden.}

Die Differentialgleichung der zu den Parametercurven $u=$ const., den Conchoiden, orthogonalen Trajectorien ist:

also:

$$
F d u+G \cdot d v=0
$$

$$
\frac{d u}{d v}+\frac{\cos ^{2} v}{p \sin v} u^{2}+\frac{2}{\operatorname{tg} v} u-\frac{p}{\sin v \cos ^{2} v}=0 .
$$

Es ist eine Riccati'sche Differentialgleichung. Weil die $z$-Axe, als Leitlinie Z, alle Conchoiden auf der Fläche rechtwinklig durchschneidet, so ist ihre Gleichung $u=\frac{p}{\cos v}$ ein Integral derselben. Um das allgemeine Integral zu finden, setzt man daher $u=\frac{p}{\cos v}+t$, dann geht die Differentialgleichung über in: 
oder :

$$
\frac{d t}{d v}-\frac{\cos ^{2} v}{p \sin v} t^{2}=0
$$

integriert:

$$
\frac{d t}{t^{2}}=\frac{1}{p} \frac{\cos ^{2} v}{\sin v} d v=\frac{1}{p}\left\{\frac{1}{\sin v}-\sin v\right\} d v \text {, }
$$

$-\frac{1}{t}=\frac{1}{p}\left\{\log \operatorname{tg} \frac{v}{2}+\cos v+\log C\right\}$ und $t=\frac{-p}{\cos v-\log \left(\operatorname{Ctg} \frac{v}{2}\right)}$.

Setzt man für $t$ seinen Wert, so ergibt sich das allgemeine Integral der Gleichung (38), oder die Parametergleichung der gesuchten orthogonalen Trajectorien :

$$
u=\frac{p \log \left(\operatorname{Ctg} \frac{v}{2}\right)}{\cos v\left(\cos v+\log \left(\operatorname{Ctg} \frac{v}{2}\right)\right)} .
$$

Durch Einsetzen in den Flächengleichungen ergeben sich die Gleichungen der Trajectorien selbst:

$$
\begin{aligned}
& x=\frac{p \sin v}{\cos v+\log \left(\operatorname{Ctg} \frac{v}{2}\right)} \\
& y=\frac{p \cos v}{\cos v+\log \left(\operatorname{Ctg} \frac{v}{2}\right)} \\
& z=-\frac{p \log \left(\operatorname{Ctg} \frac{v}{2}\right)}{\cos v\left(\cos v+\log \left(\operatorname{Ctg} \frac{v}{2}\right)\right)} .
\end{aligned}
$$

Jedem positiven Wert der Integrationsconstanten $C$ und $0 \overline{\overline{<}} v \overline{\overline{<}} \pi$ entspricht eine bestimmte Trajectorie. Für $v=0$, resp. $\pi$ wird $x=0$, $y=0, z= \pm p$, unabhängig von $C, \mathrm{~d}$. h. alle Trajectorien gehen durch die uniplanaren Doppelpunkte $S$ und $S^{\prime}$ der Fläche. Für $v= \pm \frac{\pi}{2}$ wird $x=\frac{p}{\log C}, y=0, z= \pm \infty$ und für $\operatorname{tg} \frac{v}{2}=\frac{1}{C}$ wird $x=\frac{2 p C}{C^{2}-1}, y=p$ und $z=0$, es schneiden also alle Trajectorien paarweise die Leitlinie $L$; ist $C<1$, so wird der $\mathrm{Ab}$ schnitt $x$ auf ihr von $D$ aus gerechnet negativ, für $C>1$ wird er positiv und für $C=1$ wird er unendlich. Für denjenigen Wert von $v$, für welchen der gemeinschaftliche Nenner $\cos v+\log \left(\operatorname{Ctg} \frac{v}{2}\right)=0$ ist, werden alle drei Coordinaten unendlich groß. 
Die Projection der Trajectorien auf die $(x y)$ Ebene ist:

$$
C\left(\sqrt{x^{2}+y^{2}}-y\right)=x e^{\frac{p-y}{\sqrt{x^{2}+y^{2}}}} .
$$
$y$-Axe.

Alle Curven gehen durch den Nullpunkt und berühren die Die Projection auf die $(y z)$ Ebene wird:

$$
C^{2}(z+y-p)=(z-y+p) e^{\frac{2(p-y)^{2}}{y z}} .
$$

Alle diese Curven gehen dureh den Punkt $D$ auf $L$ und durch $S$ und $S^{\prime \prime}$ auf der $z$-Axe.

Die Trajectorien $C=0$ und $C=\infty$ bestehen aus der Leitlinie $Z$ und der Erzeugenden $D S^{\prime}$, bezüglich $D S$ in der $(y z)$ Ebene.

\section{Geodätische Krümmung der Parametercurven.}

Wird eine Flächencurve $C$ senkrecht auf die Tangentialebene eines Punktes $P$ derselben projiciert, so heißt die Krümmung ihrer Projection $C^{\prime}$ im Punkte $P$ die tangentiale oder geodätische Krü m mung der Curve $C$ im Punkte $P$. Der zugehörige Krümmungsmittelpunkt $M$ der Curve $C^{\prime}$ heilt der Mittelpunkt der geodät ischen Krümmung der Curve $C$ und die Entfernung $P M$ der Radius $\rho_{g}$ der geodätischen Krämmung.

Weil die Parametercurven $v$ die geradlinigen Erzeugenden sind, so ist ihre geodiutische Krümmung $\frac{1}{P_{y}}$ stets gleich Null.

Ist die geodätische Krümmung in allen Punkten einer Curve gleich Null, so ist sie eine geodätische Linie, daher ist jede Gerade auf einer Fläche eine geodätische Linie.

Aus der Bonnet'schen Gleichung ergibt sich der Ausdruck für die geodätische Krümmung der Parameterlinien $u=$ const. (Conchoiden):

$$
\frac{1}{P_{u}}=\frac{1}{\sqrt{E G-F^{u}}}\left\{\frac{\partial}{\partial v}\left(\frac{F}{\sqrt{G}}\right)-\frac{\partial \sqrt{G}}{\partial u}\right\},
$$

also

(41) $\frac{1}{\rho_{u}}=\frac{u \cos ^{3} v(p-u \cos v)\left[2 p \sin ^{2} v-\cos ^{2} v(p-u \cos v)\right]}{\left(p^{2}-2 p u \cos ^{3} v+u^{2} \cos ^{4} v\right)^{\frac{3}{2}} \sqrt{p^{2}} \overline{\operatorname{tg}^{2} v+2(p-u \cos v)^{2}}}$.

Nun ist die geodütische Krïmmung $\frac{1}{P_{u}}=0$ : 
1. wenn $u=0$ ist: Leitlinie $L$;

2. $\quad v= \pm \frac{\pi}{2}$ ist : unendlich ferne Doppelerzeugende $G_{\infty}$;

3. $\leadsto p-u \cos v=0$ ist: Leitlinie $Z$, als Strictionslinie

4. $\quad \Rightarrow \quad 2 p \sin ^{2} v-\cos ^{2} v(p-u \cos v)=0$ oder $u=\frac{p\left(1-2 \operatorname{tg}^{2} v\right)}{\cos v}$.

Diese Gleichung stellt eine Curve auf der Fläche dar, in deren Punkten die hindurchgehenden Conchoiden die geodätische Krümmung Null haben, diese Curve ist daher die Strictionslinie des zu den Conchoiden orthogonalen Curvensystems. (Gleichung 40.)

Setzt man de Wert $u$ in den Gleichungen der Fläche ein; so erhält man die Gleichungen dieser Strictionslinie:

$$
x=2 p \operatorname{tg}^{3} v, \quad y=2 p \operatorname{tg}^{2} v, \quad z=\frac{p\left(1-2 \operatorname{tg}^{2} v\right)}{\cos v} .
$$

Die Coordinate $y$ ist stets positiv, die Curve liegt also ganz auf der positiven Seite der $(x z)$ Ebene. Für $v=0$ und $\pi$ wird $x=y=0$ und $z= \pm p$, es sind die Doppelpunkte $S$ und $S^{\prime}$. Für tg $v= \pm \frac{1}{2} \sqrt{2}$ wird $x= \pm \frac{p}{2} \sqrt{2}, y=p, z=0$ als Schnittpunkte der Curve mit Leitlinie $L$. Für $v= \pm \frac{\pi}{2}$ wird $x= \pm \infty$; $y= \pm \infty, z= \pm \infty$.

Die Projection dieser Striktionslinie auf die $(x y)$ Ebene ist die Neil'sche Parabel $y^{3}=2 p x^{2}$. Projiciert man aber alle Conchoiden, $z=u=$ constant, der Fläche senkrecht auf die $(x y)$ Ebene, so erhält man das eingangs erwähnte Conchoidensystem von der gemeinschaftlichen Asymptote $L$ und der Ort aller Wendepunkte dieses Curvensystems ist dieselbe Neil'sche Parabel $y^{3}=2 p x^{2}$.

Die Projection der Strictionslinie auf die $(y z)$ Ebene ist die Curve 3. Ordnung $z^{2}=\frac{1}{2 p}(y-p)^{2}(y+2 p)$, sie hat den Punkt $D(y=p, z=0)$ zum Doppelpunkt, und eine Schleife vom Scheitel $y=-2 p$ auf die $y$-Axe. Die Projection auf die $(x z)$ Ebene ist die Curve 6. Ordnung:

$$
4\left(x^{2}-z^{2}+p^{2}\right)^{3}=27 p^{4} x^{2} .
$$

Die Punkte $S$ and $S^{\prime}$ sind Spitzen der Curve. Hieraus folgt:

Der Ort aller Punkte der Conchoidenfläche, in welchen die geodätische Krümmung der durch sie hindurchgehenden Conchoiden $z=u=$ const. gleich Null ist, ist eine Raumeurve 6. Ordnung; welche aus zwei congruenten Zweigen besteht, welche in der Form von räumlichen Neil'schen Parabeln von den Spitzen $S$ und $S^{\prime}$ 
aus nach unten, bezüglich oben ins Unendliche gehen, wobei sie sich anf der Leitlinie $L$ durchkreuzen. Diese Curve ist die Strictionslinie der orthogonalen Trajectorien der Conchoiden und zugleich der Ort der Wendepunkte aller Conchoiden der Fläche.

In den Punkten der Erzeugenden $D S(v=0)$ wird die geodätische Krümmung $\frac{1}{\rho_{u}}=\frac{-u}{\sqrt{2}(p-u)^{2}}$. Im unendlich fernen Punkt and im Schnittpunkt $D$ dieser Geraden mit $L$ ist $\frac{1}{\rho_{u}}=0$, und im Punkte $S$ wird $\frac{1}{P_{u}}=-\infty$. In den Funkten der Erzeugenden $D S^{\prime \prime}$ durchlauft $\frac{1}{\rho_{u}}$ dieselben Werte. Die Doppelpunkte $S$ und $S^{\prime}$ sind die einzigen Punkte der Fläche, in denen $\frac{1}{\rho_{u}}$ unendlich groß wird.

Nimint man die Erzeugenden $v$ und ihre orthogonalen Trajectorien $u$ als Parametercurven an (Gleichung 35), so wird die geodätische Krümmung dieser Trajectorien:

$$
\frac{1}{p_{t}}=\frac{-\sqrt{2} \cos v(u \sqrt{2} \cos v-p)}{(u \sqrt{2} \cos v-p)^{2}+2 p^{2} \operatorname{tg}^{2} v}
$$

$\frac{1}{P_{t}}$ wird Null längs $G_{\infty}\left(v= \pm \frac{\pi}{2}\right)$ und längs der Strictionslinie $Z\left(u=\frac{p}{\sqrt{2} \cos v}\right)$.

Längs der Trajectorie $u=0$, welche die gleichseitige Hyperbel $z^{2}-x^{2}=\frac{p^{2}}{4}$ in der Ebene $y=\frac{p}{2}$ ist, wird $\frac{1}{\rho_{t}}=\frac{\sqrt{2} \cos v}{p\left(1+2 \operatorname{tg}^{2} v\right)}$, und längs der Erzeugenden $S D$ bezüglich $S D^{\prime}(v=0, \pi)$ wird $\frac{1}{\rho_{t}}=\frac{\sqrt{2}}{p \mp u \sqrt{2}}$, speciell im Punkte $D$ wird $\frac{1}{\rho_{i}}=\frac{\sqrt{2}}{p}$.

\section{Geodätische Torsion der Parametercurven.}

Unter der geodätischen Torsion in einem Punkte einer Flächencurve versteht man nach $\mathrm{B}$ on net die Torsion derjenigen geodätischen Linie, welche die Flächencurve in diesem Punkte berührt. Die Krümmungslinien der Fläche sind diejenigen Curven, welche in jedem Punkte die geodaitische Torsion Null besitzen.

Für die geodätischen Torsionen $\frac{1}{T_{u}}$ und $\frac{1}{T_{v}}$ der Parameterlinien, der Conchoiden $u=$ const. und Erzeugenden $v=$ const., gelten die folgenden Ausdrücke (Bianchi, Differentialgeometrie p. 167): 


$$
\frac{1}{T_{u}}=\frac{G D^{\prime}-F D^{\prime \prime}}{G \delta}=
$$

$$
\frac{p^{2} \sin v\left(p-u \cos v-u \cos v \sin ^{2} v\right)}{\left\{p \operatorname{tg}^{2} v+2(p-u \cos v)^{2}\right\}\left\{p^{2}-2 p u \cos ^{3} v+u^{2} \cos ^{4} v\right\}}
$$

$$
\frac{1}{T_{v}}=\frac{F D-E D^{\prime}}{E^{\delta}}=\frac{-p \sin v}{p^{2} \operatorname{tg}^{2} v+2(p-u \cos v)^{2}} .
$$

Dabei ist $\frac{1}{T_{v}}=-k^{2}$, gleich dem negativen Quadrat des Krümmungsmaßes der Fläche in demselben Punkte, denn die Erzeugenden sind Asymptotenlinien.

Für $v=0, \pi$ und $\pm \frac{\pi}{2}$ wird unabhängig von $u, \frac{1}{T_{u}}=0$ und $\frac{1}{T_{v}}=0$, d. h. die Erzengenden $D S, D S^{\prime}$ und $G_{\infty}$ sind Krümmungslinien der Fläche.

Die geodätischen Torsionen $\frac{1}{T_{u}}$ und $\frac{1}{T_{v}}$ werden gleich und entgegengesetzt.

$$
\begin{aligned}
& \text { 1. Wenn } F=0 \text { ist, dann wird } \frac{1}{T_{u}}=-\frac{1}{T_{v}}=\frac{D^{\prime}}{\sqrt{E \cdot G}}= \\
& =\frac{\sin v}{\sqrt{2\left(p^{2}-2 p u \cos ^{3} v+u^{2} \cos ^{4} v\right)}}
\end{aligned}
$$

In diesem Falle stehen die Parametercurven $u$ und $v$, also auch die sie berührenden geodätischen Linien aufeinander senkrecht. Nun ist $F=0$ für $v=0$ und $\pi$, dann wird $\frac{1}{T_{u}^{r}}=-\frac{1}{T_{v}}=0$ für jedes $u$, es sind die Erzeugenden $D S$ und $D S^{\prime}$.

2. Wenn $D=0$ und $D^{\prime \prime}=0$ ist, d. h. wenn die Parameterlinien Asymptotenlinien der Fläche sind. In diesem Falle wird $\frac{1}{T_{u}}=-\frac{1}{T_{v}}=\frac{D^{\prime}}{\delta}$.

Die Bedingung $D=0$ ist stets erfüllt, da die Parameterlinien $v$ die eine Schar der Asymptotenlinien sind, die zweite Bedingung $D^{\prime \prime}=0$ ist in folgenden drei Fällen erfüllt:

a) Wenn $u=0$ ist, $\frac{1}{T_{u}}=-\frac{1}{T_{v}}=\frac{\sin v}{p\left(2+\operatorname{tg}^{2} v\right)}$ in den Punkten der Leitlinie $L$, als Asymptotenlinie.

b) $\delta=\infty$ also $v= \pm \frac{\pi}{2}, \frac{1}{T_{u}^{\prime}}=-\frac{1}{T_{v}^{\prime}}=0$, die Doppelgerade $G_{\infty}$ als Asymptoten- und Krümmungslinie.

c) Wenn $u=\frac{p\left(1-2 \operatorname{tg}^{2} v\right)}{\cos v}$ ist, $\frac{1}{T_{u}}=-\frac{1}{T_{v}}=\frac{\cos v}{p \operatorname{tg} v\left(1+8 \operatorname{tg}^{2} v\right)}=-k^{2}$.

In den Punkten dieser Flächencurve b e $\mathbf{r}$ ü hr $\mathrm{t}$ je eine Asymptotenlinie der zweiten Schar die durch den betreffenden Punkt hindurch- 
gehende Conchoide $u$. Diese Curve ist identisch mit der im vorhergehenden Abschnitt gefundenen, welche durch die Wendepunkte aller Conchoiden hindurchgeht und längs welcher die geodätische Krümmung gleich Null ist.

In den Spitzen $S$ und $S^{\prime}$ der Curve ist die Torsion $\frac{1}{T_{u}}=-\frac{1}{T_{v}}=\infty$, von hier aus nimmt sie ab bis zu Null im Unendlichen.

Diegeodätische Torsion $\frac{1}{T_{u}}$ (Gleichung 45) wird. $\mathrm{Null}$, wenn $p-u \cos v-u \cos v \sin ^{2} v=0$ ist, oder wenn

$$
u=\frac{p}{\cos v\left(1+\sin ^{2} v\right)}
$$

Diese Curve ist daher der Ort aller solcher Punkte der Fläche, in welchen die eine Krümmungslinie die durch diesen Punkt gehende Conchoide berïhrt, während die andern sie rechtwinklig schneidet.

Setzt man diesen Wert für $u$ in den Flächengleichungen ein, so erhält man die Gleichungen dieser Curve:

$$
x=\frac{p \sin ^{2} v \operatorname{tg} v}{1+\sin ^{2} v}, \quad y=\frac{p \sin ^{2} v}{1+\sin ^{2} v}, \quad z=\frac{p}{\cos v\left(1+\sin ^{2} v\right.} .
$$

Die Coordinate $y$ wird nie negativ. Die Curve besitzt in $S(v=0)$ and $S^{\prime}(v=\pi)$ Spitzen; für $v= \pm \frac{\pi}{2}$ wird $x= \pm \infty$, $y=\frac{p}{2}, z= \pm \infty$, d. h. die Curve liegt ganz zwischen der $(x z)$ Ebene und der Ebene $y=\frac{p}{2}$.

Projection auf die $(x y)$ Ebene: $\left(x^{2}+y^{2}\right) y+(y-p) x^{2}=0$, Asymptote $y=\frac{p}{2}$, Spitze in $O$.

SProjection auf die $(y z)$ Ebene: $(p-y)^{3}-(p-2 y) z^{2}=0$, Asymptote $y=\frac{p}{2}$, Spitze in $(y=p, z=0)$.

Die Flächencurve $\frac{1}{T_{u}}=0$ besteht aus zwei congruenten, symmetrisch zur $(x y)$ und $(y z)$ Ebene liegenden Theilen, die von den Spitzen $S$ bezüglich $S^{\prime}$ aus nach oben und unten asymptotisch zur Ebene $y=\frac{p}{2}$ ins Unendliche laufen.

Die Tangentialebene an die Conchoidenfläche in einem Punkte dieser Curve hat die Gleichung:

$$
x \cos v-2 y \sin v-z \sin v \cos v+p \sin v=0,
$$


ihr Abschnitt auf der $y$-Axe ist $y=\frac{p}{2}=$ const., d. h. alle Tangentialebenen der Fläche längs der Curve $\frac{1}{T_{2}}=0$ umhüllen einen Keg el vom Scheitel $Q\left(0, \frac{p}{2}, 0\right)$. Verschiebt man das Coordinatensystem parallel nach diesem Scheitel, so wird die Gleichung dieses Kegels :

$$
\begin{gathered}
x^{\frac{2}{3}}+\left(2 y^{\prime}\right)^{\frac{2}{3}}=z^{\frac{2}{3}} \text { oder } \\
\left(z^{\prime 2}-4 y^{\prime 2}-x^{\prime 2}\right)^{3}=81 x^{\prime 2} y^{\prime 2} z^{2} .
\end{gathered}
$$

Die in $\operatorname{der}(y z)$ Ebene liegenden Erzeugenden $z^{\prime}= \pm 2 y^{\prime}$, die durch $S$ und $S^{\prime}$ gehen, sowie die in der $\left(x^{\prime} z^{\prime}\right)$ Ebene $\left(y=\frac{p}{2}\right)$ liegenden $z^{\prime}= \pm x^{\prime}$ sind R ückkehrkanten des Kegels. Die ebenen Sehnitte parallel der $(x y)$ Ebene sind Ellipsenevoluten, diejenigen parallel den zwei andern Coordinatenebenen sind Hyperbelevoluten. Speciell ist der ebene Schnitt $y_{2}^{\prime}=\frac{p}{2}$ oder $y=p$ (Asymptotenebene) die Evolute $z^{\frac{2}{3}}-x^{\frac{2}{3}}=p^{\frac{2}{3}}$ der gleichseitigen Hyperbel $z^{\prime 2}-x^{\prime 2}=\frac{p^{2}}{4}$. Diese stimmt mit der früher gefundenen Evolute (Gleichung 20a) überein, welche die Enveloppe derjenigen Geraden ist, in welchen die zur $y$-Axe parallelen Tangentialebenen der Fläche die Asymptotenébene schneiden.

\section{Ebenencoordinaten und die Reciprocalfläche.}

Die Gleichung der Tangentialebene im Punkte $(u, v)$ der Conchoidenfläche kann in der Form geschrieben werden:

$$
\frac{u \sin v \cos ^{2} v}{p(p-u \cos v)} x-\frac{\left(p-u \cos ^{3} v\right)}{p(p-u \cos v)} y-\frac{\cos v}{p} \cdot z+1=0 \text {. }
$$

Die Coefficienten von $x, y$ und $z$, nämlich:

$$
\xi=\frac{u \sin v \cos ^{2} v}{p(p-u \cos v)}, \quad \eta=-\frac{\left(p-u \cos ^{3} v\right)}{p(p-u \cos v)}, \zeta=-\frac{\cos v}{p},
$$

welche die negativen reciproken Werte der Axenabschnitte der Ebene bedeuten, heißen die E b e n e n c o ord in a t en der Tangentialebene. Durch Elimination der Parameter $u, v$ ergibt sich die Gleichung der Conchoidenfläche in Ebenencoordinaten:

$$
\xi^{2}\left(p \zeta^{2}-1\right)+(p \eta+1)^{2} \zeta^{2}=0,
$$

sie ist also eine Linienfläche 4. Classe. Setzt man $\gamma=0$, so erhält man die Enveloppe der Schnittlinien der zur $y$-Axe 
parallelen Tangentialebenen mit der $(x z)$ Ebene. Die Gleichung dieser Enveloppe in Liniencoordinaten ist:

$$
p^{2} \xi^{2} \zeta^{2}-\xi^{2}+\xi^{2}=0
$$

Diese Enveloppe haben wir schon in Abschnitt III untersucht und ihre Gleichung $(20 a)$ in rechtwinkligen Coordinaten $z^{\frac{2}{3}}-x^{\frac{2}{3}}=p^{\frac{2}{3}}$ gefunden, als Evolute der gleichseitigen Hyperbel $2^{2}-x^{2}=\frac{p^{2}}{4}$.

Betrachtet man $\xi, \gamma_{i} \zeta$ als Punkteoordinaten, so stellt die Gleichung (51) die sog. Reciprocalfläche der Conchoidenfläche dar, sie ist eine Linienfläche 4. Ordnung. Jeder Tangentialebene in einem Punkte $u, v$ der Grundfläche $F$ entspricht zufolge der Gleichung (50) ein bestimmter Punkt $\xi, \eta, \zeta$ der Reciprocalfläche $F^{\prime \prime}$ und die conjugierten Curvensysteme und Asymptotenlinien von $F$ gehen in solche von $F^{\prime \prime}$ iuber.

Verschiebt man das räumliche Coordinatensystem parallel nach dem Punkte $O^{\prime}\left(\eta=-\frac{1}{p}\right)$ auf der $y$-Axe und bezeichnet die neuen Axen und Coordinaten mit $x^{\prime}, y^{\prime}, z^{\prime}$, so wird die Gleichung (51) der Reciprokalfläche:

$$
p^{2} z^{\prime 2}\left(x^{\prime 2}+y^{\prime 2}\right)=x^{2}
$$

sie ist symmetrisch in Bezug auf alle drei neuen Coordinatenebenen. Der Nullpunkt ist uniplanarer Doppelpunkt mit der $\left(y^{\prime} z^{\prime}\right)$ Ebene als Tangentialebene.

Ein zur $(x y)$ Ebene paralleler Schnitt, $z^{\prime}=c$, besteht aus der unendlich fernen Geraden $g_{s}$ der $(x y)$ Ebene und aus dem Linienpaar $e, e^{\prime}$ (Fig. 3):

$$
x^{2}\left(1-p^{2} c^{2}\right)-p^{2} c^{2} y^{\prime 2}=0 \text { oder } y^{\prime}= \pm \frac{\sqrt{1-p^{2} c^{2}} x^{\prime}}{p^{c}}
$$

dessen Schnittpunkt auf der $z^{\prime}$-Axe liegt, dasselbe ist nur reell wenn $|c|<\frac{1}{p}$ ist. Für $c=0$ fallen sie in $\operatorname{der}(x y)$ Ebene mit der $y^{\prime}$-Axe zusammen und für $c= \pm \frac{1}{p}$ fallen sie zusammen mit zwei Parallelen zur $x^{\prime}$-Axe in der $\left(x^{\prime} z^{\prime}\right)$ Ebene im Abstand $z^{\prime}= \pm \frac{1}{p}$ von der $x^{\prime}$-Axe. Diese zwei parallelen Geraden $g$ und $g^{\prime}$ sind Doppelgerade der Fläche, zwischen den durch sie hindurchgehenden parallelen Ebenen $z^{\prime}= \pm \frac{1}{p}$ liegt die Linienfläche eingeschlossen.

Ein zur $\left(x^{\prime} z^{\prime}\right)$ Ebene paralleler Schnitt, $y^{\prime}=b$, besteht aus der Curve 4. Ordnung:

$$
p^{2} z^{\prime 2}\left(x^{2}+b^{2}\right)-x^{\prime 2}=0
$$


sie besteht aus zwei unendlichen Ästen, die sich im Nullpunkt durchschneiden, zwischen den Asymptoten $z^{\prime}= \pm \frac{1}{p}$. Der Schnitt mit der $\left(x^{\prime} z^{\prime}\right)$ Ebene selbst, $b=0$, zerfällt in die doppelte $z^{\prime}$-Axe und die zwei Geraden $z^{\prime}= \pm \frac{1}{p}$.

Der zur $\left(y^{\prime} z^{\prime}\right)$ Ebene parallele Schnitt $x^{\prime}=a$ ist die Curve 4. Ordnung:

$$
p^{2} z^{\prime 2}\left(y^{\prime 2}+a^{2}\right)-a^{2}=0 .
$$

Die Curve besteht aus zwei conchoidalen, congruenten Ästen mit den Scheiteln $z^{\prime}= \pm \frac{1}{p}$, für welche die dazwischenliegende $y^{\prime}$-Axe der Schnittebene gemeinschaftliche Asymptote ist. Der Schnitt mit der $\left(y^{\prime} z^{\prime}\right)$ Ebene selbst, $a=0$, reduciert sich auf die doppelte $y^{\prime}$ - und $z^{\prime}$-Axe.

Die Coordinaten eines Punktes der Reciprokalfläche $F^{\prime}$, welcher der Tangentialebene des Punktes $(u, v)$ der Conchoidenfläche $F$ * entspricht, sind nach den Gleichungen (50), bezogen auf das transformierte Coordinatensystem :

$$
x^{\prime}=\frac{u \sin v \cos ^{2} v}{p(p-u \cos v)}, \quad y^{\prime}=\frac{-u \cos v \sin ^{2} v}{p(p-u \cos v)}, \quad z^{\prime}=-\frac{\cos v}{p} .
$$

Jeder Geraden von $F$ als Enveloppe ihrer Tangentialebenen entspricht eine Gerade auf $F^{\prime \prime}$ als Ort der entsprechenden Punkte. Der Leitlinie $L(u=0)$ von $F$ entspricht $x^{\prime}=y^{\prime}=0, z^{\prime}=-\frac{\cos v}{p}$, d. h. die doppelte $z^{\prime}-A x e z w i s e h e n$ den Punkten $Q\left(z^{\prime}=-\frac{1}{p}\right)$ and $Q^{\prime}\left(z^{\prime}=+\frac{1}{p}\right)$ der Fläche $F^{\prime \prime}$. Der Leitlinie $Z\left(u=\frac{p^{p}}{\cos v}\right)$ der Fläche $F$ entspricht die unendlich ferne Doppelgerade $g_{\infty}$ der $\left(x^{\prime} y^{\prime}\right)$ Ebene in $F^{\prime}$.

Der Erzeugenden $v$ von $F^{\prime}$ entspricht die Erzeugende $y^{\prime}=-\operatorname{tg} v \cdot x^{\prime}, z^{\prime}=-\frac{\cos v}{p}$ der Reciprokalfläche $F^{\prime \prime}$, welche parallel ist der $\left(x^{\prime} y^{\prime}\right)$ Ebene und die $z^{\prime}$-Axe schneidet. Durch jeden Punkt der $z^{\prime}$-Axe gehen zwei solche Erzengende $( \pm v)$, symmetrisch zur $\left(x^{\prime} z^{\prime}\right)$ Ebene.

Für $v=0$ fallen sie zusammen in $y^{\prime}=0, z^{\prime}=-\frac{1}{p}$, d. h. der Erzeugenden $D S$ von $F$ entspricht die Doppelgerade $g^{\prime}$ in der $\left(x^{\prime} z^{\prime}\right)$ Ebene parallel zur $x^{\prime}$-Axe in $F^{\prime \prime}$. Bei positiv bezüglich negatir wachsendem $v$ wächst auch der Winkel zwischen den entsprechenden reciproken Erzeugenden und für $v= \pm \frac{\pi}{2}$ wird ihr Winkel gleich 
$180^{\circ}$, sie fallen in der $y^{\prime}$-Axe zusammen. Bei weiter positiv und negativ wachsendem $v$ treten die reciproken Erzeugenden wieder auseinander, und für $v= \pm \pi$ wird ihr Winkel $=360^{\circ}$, sie fallen zusammen in der Doppelgeraden $\left(y^{\prime}=0, z^{\prime}=+\frac{1}{p}\right)$ in $\operatorname{der}\left(x^{\prime} z^{\prime}\right)$ Ebene, diese entspricht der Erzeugenden $D S^{\prime}$ von $F$. Die reciproken Erzeugenden sind ferner parweise zu einander parallel und parallel $\operatorname{der}\left(x^{\prime} y^{\prime}\right)$ Ebene, in der Eibene $y^{\prime}=-\operatorname{tg} v \cdot x^{\prime}$ liegend, entsprechend den Werten $v$ und $\pi+v$.

Die Parametercurven $u=$ const, auf $F^{\prime \prime}$ sind Raumcurven 6. Ordnung, die alle in $O^{\prime}$ einen Doppelpunkt haben und nur symmetrisch sind zur $\left(y^{\prime} z^{\prime}\right)$ Ebene.

Zusammengefasst finden wir:

Die Reciprokalfläche der Conchoidenfläche ist eine Linienfläche 4. Ordnung, die symmetrisch ist zu allen drei Coordinatenebenen und ganz zwischen den zwei parallelen Ebenen $z^{\prime}=\mp \frac{1}{p}$ verläuft. Ihre geradlinigen Erzeugenden sind alle parallel der $\left(x^{\prime} y^{\prime}\right)$ Ebene und schneiden sich paarweise auf der $z^{\prime}$-Axe und auf der unendlich fernen Geraden $g_{\infty}$ der $\left(x^{\prime} y^{\prime}\right)$ Ebene, denn die Erzeugenden sind noch paarweise parallel in Ebenen durch die $z^{\prime}$-Axe. Die Fläche besitzt fünf Doppelgerade: die $y^{\prime}$ und $z^{\prime}$-Axe, $g_{\infty}$ und die parallelen Geraden $z^{\prime}= \pm \frac{1}{p}$ in der $\left(x^{\prime} z^{\prime}\right)$ Ebene, $g$ und $g^{\prime}$. (Fig. 3.)

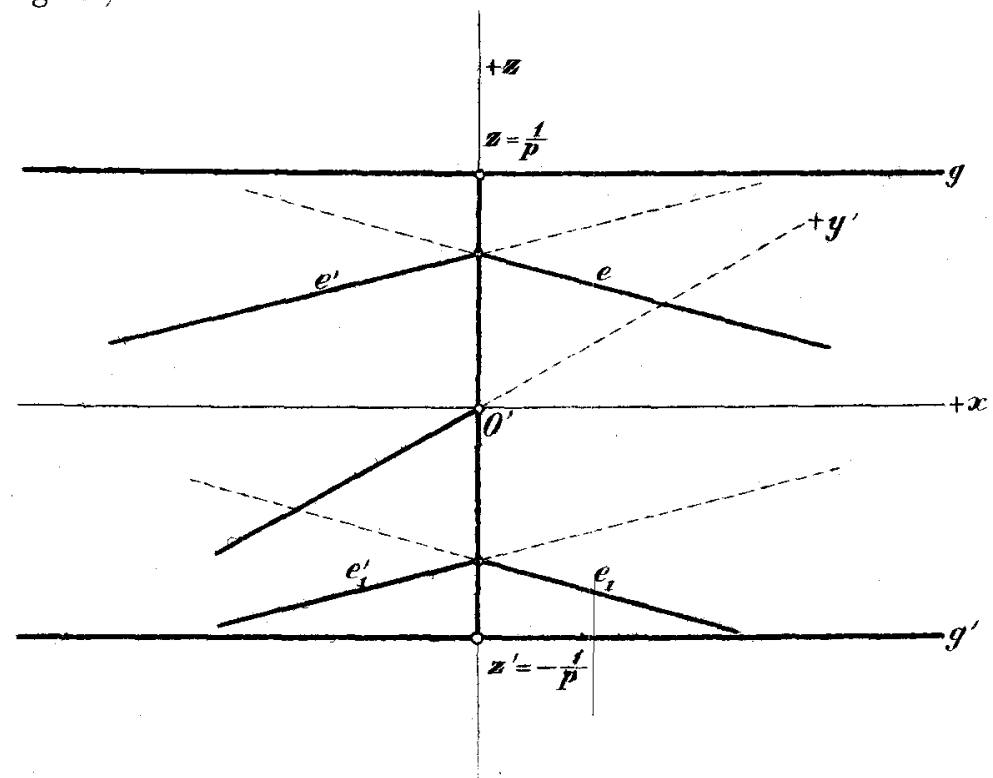

Fig. 3. Reciprokaltäche. 
Zwischen diesen äulersten Geraden $g$ und $g^{\prime}$ erstreckt sich die Fläche in der Form von zwei unendlich langen Windmühlenflügeln, von denen der eine rechts, der andere links um die $z^{\prime}$-Axe gewunden ist; diese durchsetzen sich längs der $y^{\prime}$-Axe und treffen in den Geraden $g$ and $g^{\prime}$ zusammen. Die Drehung jedes Flügels beträgt $180^{\circ}$.

\section{Die Kernfläche.}

Aus der gleich Null gesetzten Hes se'schen Determinante der Conchoidenfläche èrgibt sich die Gleichung ihrer Kernfläche:

$$
(y-p)^{2}\left(x^{2}+y^{2}\right)-z^{2}\left(y^{2}-3 x^{2}\right)=0 .
$$

Die Fläche ist eine Linienfläche 4. Ordnung, die symmetrisch zur $(x y)$ und $(y z)$ Ebene liegt. Die $(x y)$ Ebene schneidet die Kernfläche in der Doppelgeraden $L(y=p)$ und im conjugierten Punkt $O$, die $(y z)$ Ebene in der doppelten $z$-Axe Doppelgerade $Z$, und in den beiden Geraden $y \pm z=p$, welche als Erzengende $D S$ und $D S^{\prime}$ der Conchoidenfläche angehören. Die $(x z)$ Ebene schneidet in der Doppelgeraden $Z$ und den imaginären Geraden $3 z^{2}+p^{2}=0$.

Eine durch die $z$-Axe gelegte Ebene $y=\operatorname{tg} a \cdot x$, welche durch Drehung um die $z$-Axe zur $(x z)$ Ebene gemacht wird, schneidet die Kernfläche in den beiden Geraden:

$$
z= \pm \frac{1}{\sqrt{1-4 \cos ^{2} a}}\left(x^{\prime} \sin \alpha-p\right) \text {. }
$$

Die beiden Geraden sind nur reell, wenn $60^{\circ}<\alpha<120^{\circ}$ ist, sie gehen durch denselben Punkt der $x^{\prime}$-Axe im Abstand $x^{\prime}=\frac{p}{\sin \alpha}$ von $O$, welcher ein Punkt von $L$ im Abstand $x=p \operatorname{cotg} \alpha$ von $D$ ist und schneiden $Z$ im Abstand $z=\frac{ \pm p}{\sqrt{1-4 \cos ^{2} \alpha}}$ von $O$. Für $a=60^{\circ}$ und $120^{\circ}$ wird $z= \pm \infty$ und für $z=90^{\circ}$ wird $z= \pm p$, Schnittpunkte $S$ und $S^{\prime}$, es ist dies der absolut kleinste Wert, den $z$ annehmen kann; zwischen $S$ und $S^{\prime}$ wird die Dopgelgerade $Z$ von keinen Geraden geschnitten, die Strecke $S S^{\prime}$ derselben ist, wio bei der Conchoidenfläche, isolierte Doppelgerade. Die Punkte $S$ und $S^{r}$ sind ebenfalls uniplanare Doppelpunkte der Kernfläche, mit der $(y z)$ Ebene als Tangentialebene.

In den Grenzfüllen $\alpha=60^{\circ}$ und $\alpha=120^{\circ}$ fallen die beiden Geraden zusammen und werden parallel zur $z$-Axe, diese beiden Geraden $l$ and $l^{\prime}$, welche die Leitlinie $L$ im Abstand $x^{-}= \pm \frac{p}{\sqrt{3}}$ von $D$ in den Punkten $F, F^{\prime}$ schneiden (Fig. 4), sind die äußersten Erzeugenden, welche $L$ schneiden, längs derselben berühren be- 
züglich die Ebenen $y= \pm \sqrt{3} x$ die Kernfläche. Dem Winkel $\alpha=90^{\circ}$ entsprechen die zwei Geraden $D S$ und $D S^{\prime}$ in der $(y z)$ Ebene.

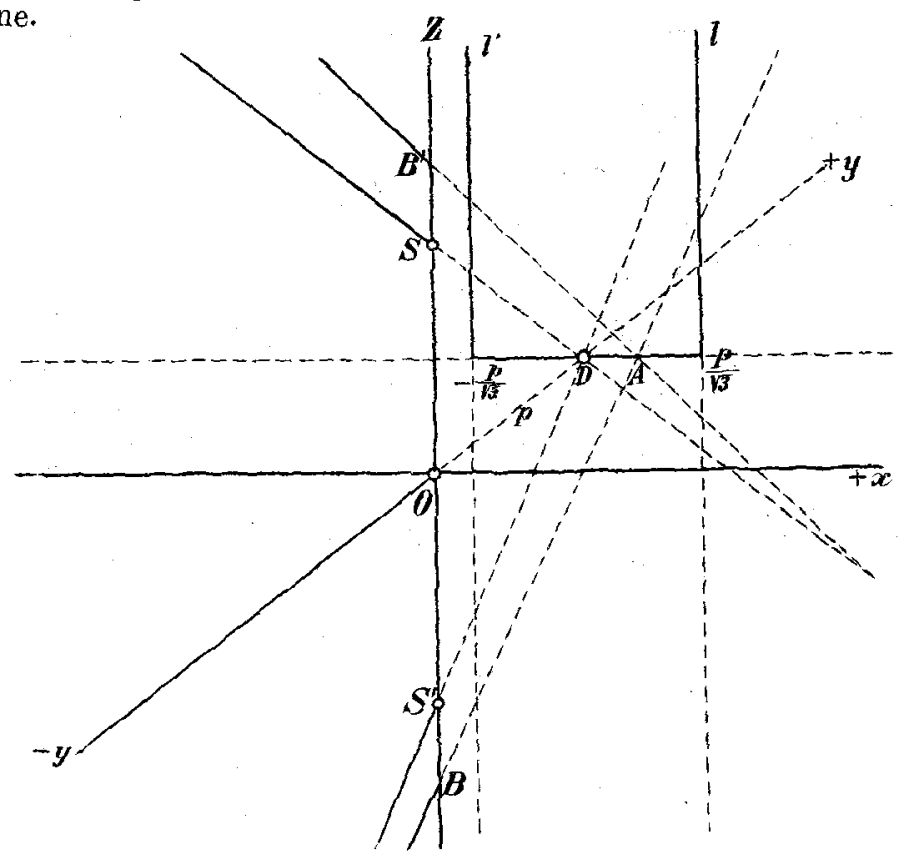

Fig. 4. Kernfläche.

Wir betrachten nun Schnitte der Kernfäche mit Ebenen, die durch die Leitlinie $L$ gehen. Macht man durch Parallelverschiebung $L$ zur $x$-Axe, so dass die Asymptotenebene der Conchoidenfläche zur $(x z)$ Ebene wird, so ist die Gleichung einer Schnittebene $y=\operatorname{tang} \beta . z$, wo $\beta$ ihr Neigungswinkel gegen die Asymptotenebene ist. Macht man diese durch Drehung des Coordinatensystems zur $\left(x^{\prime} z^{\prime}\right)$ Ebene, so wird die Gleichung der Schnitteurve:

$$
x^{\prime}= \pm \sqrt{\frac{1-\operatorname{tg}^{2} \beta}{3+\operatorname{tg}^{2} \beta}}\left(z^{\prime} \sin \beta+p\right) \text {. }
$$

Der Schnitt besteht aus zwei Geraden durch den Punkt $z^{\prime}=\frac{-p}{\sin \beta}$ der $z^{\prime}$-Axe, welcher der Schnittpunkt der Schnittebene mit der ursprünglichen $z$-Axe, $z=-p \operatorname{cotg} \beta$ ist; durch diesen Punkt gehen auch die beiden in dieser Ebene liegenden Erzeugenden der Conchoidenfläche, ihre Fußpunkte in $L$ dagegen, $x^{\prime}= \pm \sqrt{\frac{\cos 2 \beta}{1+2 \cos ^{2} \beta}} p$, sind näher an $D$, als diejenigen der Conchoidenfläche, ihr Maximalabstand von $D$ tritt ein für $\beta=0$, nämlich 
$x^{\prime}= \pm \frac{p}{\sqrt{3}}$, es sind dies die, Fußpunkte $F$ und $F^{\prime \prime}$ der zu $Z$ parallelen Erzeugenden $l$ und $l^{\prime}$ der Kernfläche. Die beiden Schnittgeraden sind ïberhaupt nur dann reell, wenn $\beta$ absolut $<45^{\circ}, d$. $h$. wenn die Schnittebenegegen die Asymptotenebene beiderseits weniger als $45^{\circ}$ geneigt ist, wie dies auch bei der Conchoidenfläche der Fall ist. Den Werten $\beta= \pm 45^{\circ}$ entsprechen die Erzeugenden $D S$ und $D S^{\prime}$.

Eine zur $(x y)$ Ebene parallele Ebene $z=c$ schneidet die Kernfläche in der Curve 4. Ordnung:

$$
(y-p)^{2}\left(x^{2}+y^{2}\right)=c^{2}\left(y^{2}-3 x^{2}\right) \text {. }
$$

Denkt man sich alle diese Schnittcurven, die einem ver. änderlichen Abstand $c$ entsprechen, orthogonal auf die $(x y)$ Ebene projiciert, wobei sie unverändert bleiben, so berühren alle Curven die zwei festen Geraden $y= \pm \sqrt{3} . x$ in den festen Punkten $T$ und $T^{\prime \prime}\left(x= \pm \frac{p}{\sqrt{3}}, y=p\right)$ auf der Leitlinie $L$ (Fig. 5). Die Schnitt-

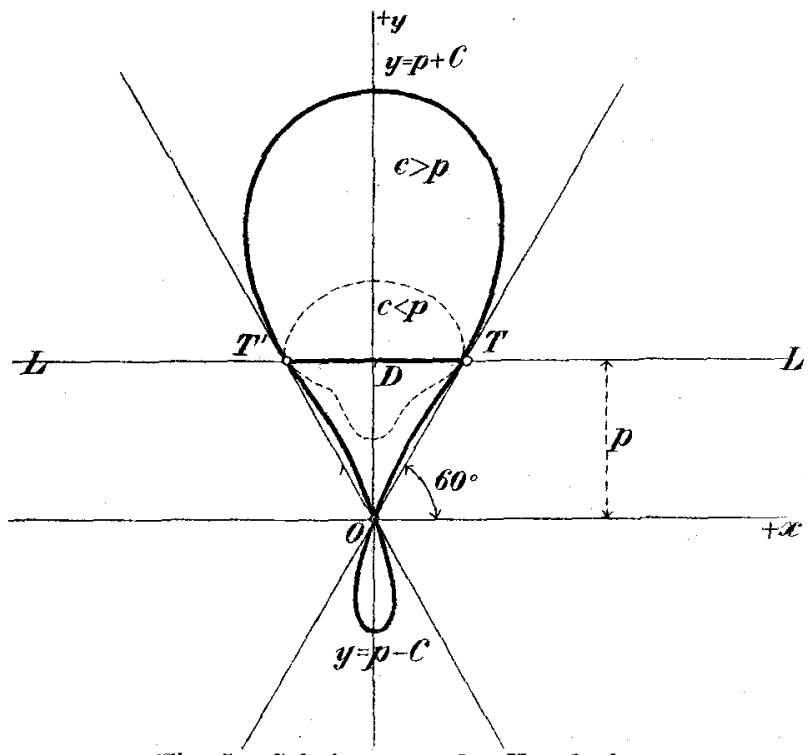

Fig. 5. Schnitt $z=c$ der Kernfäche.

punkte mit der $y$-Axe sind $y=p \pm c$, der Nullpunkt ist Doppelpunkt mit den Tangenten $y= \pm \sqrt{\frac{3 c^{2}+p^{2}}{c^{2}--p^{2}}} \cdot x$. Dabei sind folgende
Fälle zu unterscheiden:

1. Für $c<p$ besteht die Curve aus einem geschlossenen Blatte zwischen $T$ und $T^{\prime}$, Scheitel $y=p \pm c$ und dem conjugierten Punkt 0. (Fig. 5). 
2. Für $c=0$ reduciert sich das Blatt auf die Strecke $T T^{\prime \prime}$ der Leitlinie $I$.

3. Für $c=p$ besitzt das Blatt im Nullpunkt eine Spitze, mit der $y$-Axe als Rückkehrtangente.

4. Für $c>p$ besteht die Curve aus zwei ungleichen Schleifen mit Knotenpunkt in $O$ and den Scheiteln $x=0, y=p \pm c$.

5. Für $c=\infty$ zerfällt die Curve in das Geradenpaar $y= \pm \sqrt{3} . x$ und in einen Kreis von unendlich großem Radius, der um den Nullpunkt beschrieben ist. In diesen Linien schneidet die Kernfläche die unendlich ferne Ebene $z=\infty$, und zwar gehören nur diejenigen Kreisbogen der Schnittlinie an, welche zwischen den Schnittgeraden $y= \pm \sqrt{3} . x$ in denjenigen Winkelräumen liegen, durch welche die $(y z)$ Ebene geht.

Mit Hilfe dieser Schnitte kann man sich leicht ein Bild der Kernfläche machen.

Der Leitkegel der Kernfläche hat zur Gleichung:

$$
y^{2}\left(x^{2}+y^{2}\right)=z^{2}\left(y^{2}-3 x^{2}\right) \text {. }
$$

Die zur $(x y)$ Ebene parallelen ebenen Schnitte, $z=c$, sind lemniscatenförmige Curven, mit congruenten Schleifen, deren Scheitel auf der $y$-Axe in $y= \pm c$ liegen, der Nullpunkt auf der $z$-Axe ist doppelter Inflexionsknoten mit den Tangenten $y= \pm \sqrt{3} \cdot x$, welche für alle Schnittcurven dieselbe Lage haben. Der Kegel besteht aus zwei congruenten Mänteln, die längs der Doppelkante $Z$ zusammenhängen, die Tangentialebenen längs derselben sind $y= \pm \sqrt{3} . x$. Die in der $(y z)$ Ebene liegenden Erzeugenden des Kegels sind $z= \pm y$, die eine dem einen, die zweite dem andern Kegelmantel angehörend. Der Leitkegel schneidet die unendlich ferne Ebene $z=-\infty$ in denselben zwei Geraden $y= \pm \sqrt{3} . x$, nebst zugehörigen Kreisbogen von unendlich großem Radius wie die Kernfläche selbst.

Hiernach ergibt sich folgende Erzeugung der Kernfläche der Conchoidenfläche als Linienfläche 4. Ordnung:

Die Kernfläche wird erzeugt durch Bewegung einer Geraden, die stets drei ebene Leitlinien schneidet. Zwei derselben sind die beiden zu einander senkrechten und windschiefen Geraden $L$ und $Z$, welche auch Leitlinien der Conchoidenfläche sind, während die dritte, in der zu $Z$ senkrecht stehenden unendlich fernen Ebene liegende, aus zwei Geraden besteht, die sich im unendlich fernen Punkt von $Z$ unter dem Winkel $60^{\circ}$ schneiden und aus zwei quasi ihre Enden verbindenden Kreisbogen von unendlich großem Radius.

Der Durchschnitt der Conchoidenfäche mit ihrer Kernfläche ist eine Raumcurve, welche der Ort der parabolischen oder Wendepunkte der gegebenen Fläche ist; längs derselben ist das Krümmungsmaß $k=0$. Ist nun $f=0$ die Gleichung der 
Conchoidenfläche, so lässt sich die Gleichung ihrer Kernfläche $f+3 x^{2} z^{2}=0$ schreiben. Die Durchschnittscurve der beiden Flächen zerfällt also in ebene Curven, die in der $(y z)$ und $(x y)$ Ebene liegen, diese bestehen aus den Doppelgeraden $L$ und $Z$ und den beiden Erzeugenden $D S$ und $D S^{\prime}$ in der $(y z)$ Ebene, welche beiden Flächen angehören. Die letzteren sind als parabolische Linien der Conchoidenfläche zu betrachten, wie früher gefunden, ist das Krümmungsmaf längs derselben Null.

\section{Complanation der Conchoidenfläche.}

Weil alle Erzeugenden der Fläche die beiden Leitlinien $L(u=0)$ und $Z\left(u=\frac{p}{\cos v}\right)$ schneiden, so bilden die in $\operatorname{der}(y z)$ Ebene liegende Erzeugende $D S(v=0)$ und eine beliebige Erzeugende $v$ mit $L$ und $Z$ ein windschiefes Viereck $D S A B$ (Fig. 6), dessen Seiten ein geschlossenes Stück der Oberfläche begrenzen.

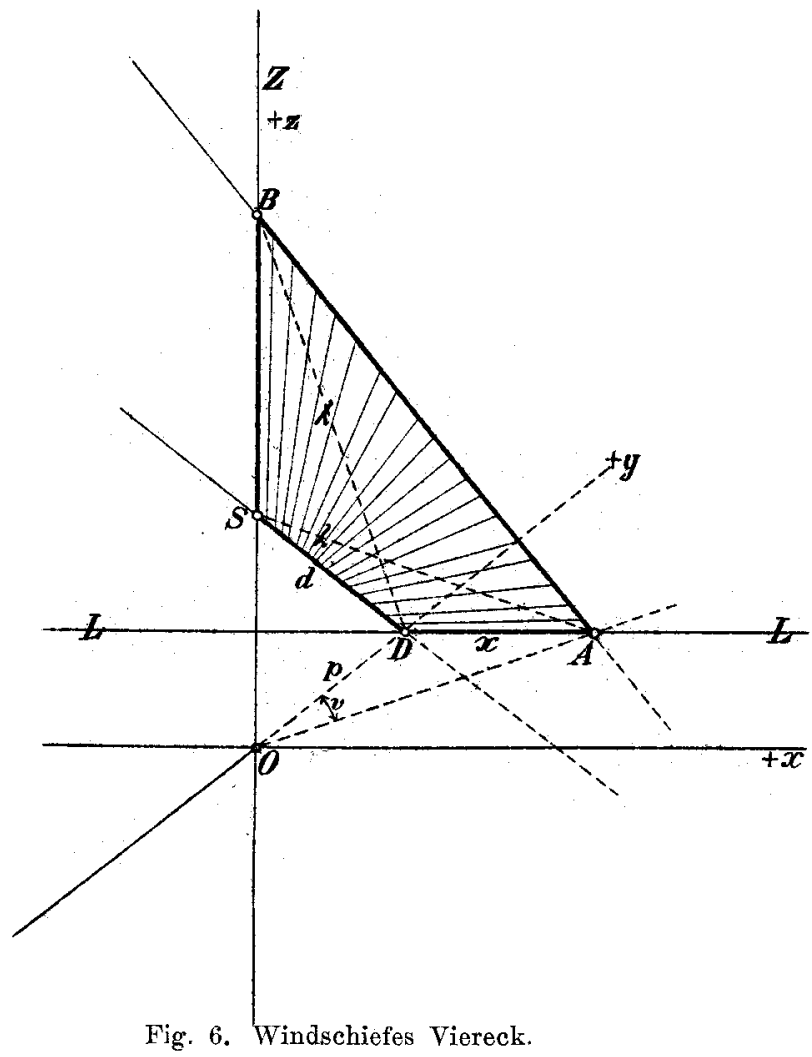


Wir wollen den Inhalt dieses Flächenstückes bestimmen. Das Flächenelemeint ist:

$$
\begin{gathered}
d F=\sqrt{E G-F^{2}} \cdot d u d v= \\
=\frac{\sqrt{2}}{\cos v} \sqrt{u^{2} \cos ^{2} v-2 p u \cos v+\frac{p^{2}}{2}\left(2+\operatorname{tg}^{2} v\right)} d u d v .
\end{gathered}
$$

Um den Inhalt des windschiefen Vierecks $D S A B$ zu erhalten, ist nach $u$ von $u=0$ bis $u=\frac{p}{\cos v}$ und nach $v$ von $v=0$ bis $v$ $\mathrm{zu}$ integrieren, also

$$
F=\int_{0}^{v} \frac{\sqrt{2}}{\cos v} d v \int_{0}^{p} \sqrt{u^{2} \cos ^{2} v-2 p u \cos v+\frac{p^{2}}{2}\left(2+\operatorname{tg}^{2} v\right)} d u .
$$

Nun ist das innere Integral:

$$
\begin{aligned}
\int_{0}^{\frac{p}{\cos v}}=\left[\frac{(u \cos v-p)}{2 \cos v} \sqrt{(u \cos v-p)^{2}+\frac{p^{2}}{2} \operatorname{tg}^{2} v+}\right. \\
\left.+\frac{p^{2} \operatorname{tg}^{2} v}{4 \cos v} \log \cos v\{(u \cos v-p)+V \bar{R}\}\right]_{0}^{\frac{p}{\cos v}},
\end{aligned}
$$

wo $R$ den gleichen Ausdruck wie unter dem ersten Wurzelzeichen bedeutet. Setzt man die Grenzen ein, so wird:

$$
\int_{0}^{\frac{p}{\cos r}}=\frac{p^{2}}{2 \sqrt{2} \cos v}\left\{\frac{\operatorname{tg}^{2} v}{\sqrt{2}} \log \frac{\left(\sqrt{2+\operatorname{tg}^{2} v}+\sqrt{2}\right)}{\operatorname{tg} v}+\sqrt{2+\operatorname{tg}^{2} v}\right\},
$$

somit:

$$
F=\frac{p^{2}}{2 \sqrt{2}} \int_{0}^{n} \operatorname{tg}^{2} v \log \left(\frac{\sqrt{2+\operatorname{tg}^{2} v}+\sqrt{2}}{\operatorname{tg} v}\right) \cdot \frac{d v}{\cos ^{2} v}+\frac{p^{2}}{2} \int_{0}^{v} \sqrt{2+\operatorname{tg}^{2} v} \frac{d v}{\cos ^{2} v} .
$$

Wir substituieren $\operatorname{tg} v=\sqrt{2} . t, \frac{d v}{\cos ^{2} v}=\sqrt{2} \cdot d t$ und integrieren unbestimmt:

$$
J=p^{2} \int t^{2} \log \left(\frac{\sqrt{1+t^{2}}+1}{t}\right) d t+p^{2} \int \sqrt{1+t^{2}} d t=p^{2}\left(J_{1}+J_{2}\right) .
$$

Nun ergibt sich durch partielle Integration:

$$
J_{1}=\frac{1}{3} \int \log \left(\frac{\sqrt{1+t^{2}}+1}{t}\right) d t^{3}=\frac{1}{3} t^{3} \log \left(\frac{\sqrt{1+t^{2}}+1}{t}\right)+\frac{1}{3} \int \frac{t^{2}}{\sqrt{1+t^{2}}} d t
$$


$J_{1}=\frac{1}{3}\left\{t^{3} \log \left(\frac{\sqrt{1+t^{2}}+1}{t}\right)+\frac{t}{2} \sqrt{1+t^{2}}-\frac{1}{2} \log \left(t+\sqrt{1+t^{2}}\right)\right\}$.

Ferner :

$J_{2}=\int \sqrt{1+t^{2}} d t=\frac{t}{2} \sqrt{1+t^{2}}+\frac{1}{2} \log \left(t+\sqrt{1+t^{2}}\right)$,

also

$J=\frac{p^{2}}{3}\left\{t^{3} \log \frac{\sqrt{1+t^{2}}+1}{t}+\log \left(t+\sqrt{1+t^{2}}\right)+2 t \sqrt{1+t^{2}}\right\}$.

Substituiert man rückwärts $t=\frac{1}{\sqrt{2}} \operatorname{tg} v$ und setzt die Grenzen ein, so verschwindet der ganze Ausdruck an der untern Grenze and man erhält den Inhalt des windschiefen Vierecks $D S A B$ :

$$
\begin{aligned}
F=\frac{p^{2}}{3}\left\{\frac{\operatorname{tg}^{3} v}{2 \sqrt{2}} \log \left(\frac{\sqrt{2}+\sqrt{2+\operatorname{tg}^{2} v}}{\operatorname{tg} v}\right)+\right. \\
+\log \left(\frac{\left.\left.\operatorname{tg} v+\frac{\sqrt{2}+\operatorname{tg}^{2} v}{\sqrt{2}}\right)+\operatorname{tg} v \sqrt{2+\operatorname{tg}^{2} v}\right\}}{}\right.
\end{aligned}
$$

als Function des Parameters $v$ der veränderlichen Seite $A B$ des Vierecks. Speciell wird

für $v=\frac{\pi}{4}: \quad F=\frac{p^{2}}{3}\left\{\frac{1}{2 \sqrt{2}} \log (\sqrt{2}+\sqrt{3})+\log \frac{1+\sqrt{3}}{\sqrt{2}}+\sqrt{3}\right\}$ für tg $v=\sqrt{2}: F=\frac{2 p^{2}}{3}\{\log (1+\sqrt{2})+\sqrt{2}\}$.

Der Ausdruck für $F$ lässt sich noch auf andere Form bringen. Im windschiefen Viereck $A B S D$ (Fig. 6) ist:

Seite $D S=d=\sqrt{O D^{2}+O S^{2}}=p \sqrt{2}=\mathrm{constant}$

Seite $D A=x=p \operatorname{tang} v$

Diagonale $A S=\lambda=\sqrt{O A^{2}+O S^{2}}=\sqrt{\frac{p^{2}}{\cos ^{2} v}+p^{2}}=p \sqrt{2+\operatorname{tg}^{2} v}$

Diagonale $D B=\lambda^{\prime}=\sqrt{O B^{2}+O D^{2}}=\sqrt{\frac{p^{2}}{\cos ^{2} v}+p^{2}}=p \sqrt{2+\operatorname{tg}^{2} v}$,

also ist $\lambda^{\prime}=\lambda$, d. h. jedes dieser windschiefen Vierecke hat gleich lange Diagonalen, folglich sind die Schnittpunkte jeder Erzeugenden der Fläche mit den Leitlinien $L$ und $Z$ sowohl gleich weit vom Nullpunkt $O$, als auch von den Punkten $S$ und $D$ entfernt, in welchen zwei Punkten die Erzeugende $v=0 Z$ und $L$ schneidet. 
Führt man die Werte $d, x$ und $\lambda$ in der Inhaltsformel (61) ein, so wird dieselbe:

$$
F=\frac{1}{3}\left\{\frac{x^{3}}{2 d} \log \left(\frac{d+\lambda}{x}\right)+p^{2} \log \left(\frac{x+\lambda}{d}\right)+\lambda x\right\} .
$$

Weil nun $x=\sqrt{\lambda^{2}-D S^{2}}=\sqrt{\lambda^{2}-d^{2}}$ ist, so wird auch :

(63) $F=\frac{1}{3}\left\{\frac{\left(\lambda^{2}-d^{2}\right)^{\frac{3}{2}}}{4 d} \log \frac{\lambda+d}{\lambda-d}+p^{2} \log \frac{\sqrt{\lambda^{2}}-\overline{d^{2}}+\lambda}{d}+\lambda \sqrt{\lambda^{2}-d^{2}}\right\}$, womit der Inhalt des windschiefen Vierecks durch die constante Seite $d$ und die veränderliche Diagonale $\lambda$ ausgedrückt ist.

Die Seite $A B$ des Vierecks, $\mathrm{d}$, h. die Länge der Erzeugenden $v$ zwischen den Leitlinien $L$ und $Z$ findet man aus dem gleichschenklig rechtwinkligen Dreieck $O A B$, in welchem $O B=O A=\frac{p}{\cos v}$ ist, nämlich $A B=r=\frac{p \sqrt{2}}{\cos v}$. Diese Länge, sowie die Abschnitte $O A=$ $=O B$ lassen sich leicht für jeden Wert von $v$ construieren. Man zieht in einem rechtwinkligen Coordinatensystem die zur $x$-Axe parallelen Geraden $y=p$ (Leitlinie $L$ ) und $y=p \sqrt{2}$ und legt von $O$ aus einen Strahl unter dem Winkel $v$ zur $y$-Axe, welcher die beiden Parallelen in $A$ bezüglich $Q$ schneidet, dann ist $O A=\frac{p}{\cos v}$ und $O Q=r=\frac{p \sqrt{2}}{\cos v}$.

Die Coordinaten der Mitte der Erzeugenden $A B$ sind:

$$
x=\frac{p}{2} \operatorname{tg} v, \quad y=\frac{p}{2}=\text { constant }, \quad z=\frac{p}{2 \cos v} .
$$

Durch Elimination von $v$ folgt: $z^{2}-x^{2}=\frac{p^{2}}{4}, \mathrm{~d}$. h. der Ort der Mitten der zwischen den Leitlinien $L$ und $Z$ liegenden Strecken sämmtlicher Erzeugenden der Conchoidenfläche ist diejenige gle ichseitige Hyperbel in der Ebene $y=\frac{p}{2}$ (Gleich. 7), in welcher der Leitkegel die Fläche durchschneidet. 